\title{
Flow hydrodynamics of the mixing layer in consecutive vegetated groyne fields
}

\author{
Cite as: Phys. Fluids 32, 065110 (2020); doi: 10.1063/5.0006317 \\ Submitted: 29 February 2020 - Accepted: 2 June 2020 • \\ Published Online: 19 June 2020
}

\author{
Ke Xiang (向珂), ${ }^{\top}$ Zhonghua Yang (杨中华), ${ }^{1, a)}$ (D) Shiqiang Wu (吴时强), ${ }^{2}$ Wei Gao (高伟), ${ }^{3}$ (D) Dan Li (李丹), \\ and Qiong Li (李琼)
}

\begin{abstract}
AFFILIATIONS
${ }^{1}$ State Key Laboratory of Water Resources and Hydropower Engineering Science, Wuhan University, Wuhan 430072, China

${ }^{2}$ State Key Laboratory of Hydrology-Water Resources and Hydraulic Engineering, Nanjing Hydraulic Research Institute, Nanjing 210029, China

${ }^{3}$ Mechanical Engineering, Physical Science and Engineering Division, King Abdullah University of Science and Technology, Thuwal 23955-6900, Saudi Arabia
\end{abstract}

a) Author to whom correspondence should be addressed: yzh@whu.edu.ch

\begin{abstract}
In recent years, increasing attention has been paid to the ecological role of groyne fields as habitats for aquatic vegetation; however, knowledge on interactions between vegetation and recirculating flow is still lacking, especially vegetation effects on large-scale coherent structures in the mixing layer, which control the mass exchange between the side-cavity and the main channel. In this paper, the hydrodynamics of the mixing layer in straight open channels without sediments in the flow, with consecutive groyne fields, of different vegetation densities, is investigated both experimentally through particle image velocimetry and numerically through large eddy simulation. The results show that the presence of plants rearranges the circulation systems in the groyne field, namely, from double gyres to a single gyre. With an increase in the vegetation density, the exchange coefficient between the cavity and the main channel gradually decreases. Note that the exchange rate is calculated from a newly proposed exchange layer, which is located away from the groyne tip. Based on the analysis of the Kelvin-Helmholtz eddies along the shear layer, a phenomenological model is proposed for the evolution of coherent structures and the variations in flow hydrodynamics associated with these eddies. Compared to the non-vegetation case, the presence of vegetation could suppress the evolution of coherent eddies in the mixing layer, with a consequent effect on the flow hydrodynamics around the interface.
\end{abstract}

Published under license by AIP Publishing. https://doi.org/10.1063/5.0006317

\section{INTRODUCTION}

For many years, groynes have been constructed along riverbanks for a variety of engineering purposes, such as improving navigation capacity, protecting banks from erosion, and promoting sediment transport in the main channel. ${ }^{1-3}$ In recent years, the ecological function of the lateral cavities between successive groynes has received an increasing amount of attention. In these cavities, the velocity magnitude is generally reduced to $25 \%-30 \%$ of the mainstream velocity, and this can greatly accelerate sediment deposition and increase the residence time of organic matters, thus creating a favorable habitat for aquatic organisms. ${ }^{4-10}$

By considering the value of the aspect ratio $\kappa=W / L$, where $W$ is the length of the groyne and $L$ is the distance between successive groynes, the circulation pattern in the side-cavity can be determined. ${ }^{4,11-15}$ When $\kappa>0.5$, only a single circulation exists in the groyne field. When $\kappa<0.5$, there are two circulation structures in the side-cavity: a primary gyre occupying most of the cavity and a small secondary gyre located in the upstream corner. Typically, the horizontal dimensions of the groyne field are much larger than the corresponding mean water depth. Thus, the recirculating flow in the side-cavity can be regarded as a two-dimensional motion, exhibiting no variation with depth. ${ }^{16}$ However, the flow along the interface between the cavity and the main channel is much more complicated and possesses a pronounced three-dimensional character: for example, the fluid penetrates into the embayment region mostly via the bottom layer of the downstream interface and escapes from the embayment primarily through the top layer of the upstream 
interface. ${ }^{16-18}$ Essentially, the complexity of the flow in the mixing layer can be ascribed to the shedding of Kelvin-Helmholtz eddies from the tip of the groyne with a master frequency, which then randomly merge with adjacent eddies, thus resulting in the formation of large-scale coherent structures along the interface. ${ }^{19-25}$ Such largescale coherent eddies play an important role in promoting mass exchange between the groyne field and the main channel through the generation of periodic or quasiperiodic alternating positive and negative fluctuations of the transverse velocity. ${ }^{1}$

The favorable conditions in groyne fields can promote the establishment of plants such as reeds that can help to reduce the eutrophication level of a river by absorbing dissolved nutrients and by enhancing the deposition of sediments (possibly contaminated with heavy metals) as a result of decreased circulation velocity, and this is beneficial to restore the stream corridor. ${ }^{34-44}$ Therefore, it is important to investigate the effect of vegetation on the flow hydrodynamics in the groyne field. It has been shown that the presence of vegetation can rearrange circulation structures (e.g., from doublecirculation flow into slow backwater flow), reduce the turbulent intensity in the cavity, and affect momentum and mass exchange across the interface. ${ }^{18,37-39,45-47}$ Vegetated side-cavities in previous studies can be summarized into two categories: the first kind, i.e., only a single vegetated embayment exists in the river ${ }^{18,45}$ and the second kind, i.e., multiple groyne fields are constructed along the riverside, and vegetation is assumed to be present in a single cavity. $^{37,38}$ As for the presence of vegetation in consecutive cavities, here referred to as the third kind, it has not been investigated before. Compared to the first kind, the streamwise distance in the third kind is long enough to make the mixing layer reach a fully developed state. For the second kind, the shear layer upstream of the focused region is not affected by vegetation due to the absence of plants in upstream cavities, which is different from the third kind. As the downstream shear layer is generally affected by the upstream ones, the difference in upstream shear layers between these two kinds could influence the flow hydrodynamics in the study region. Sukhodolov et al. ${ }^{37,38}$ experimentally investigated the shear layer structure of the second kind configuration and stated that further research of the third kind should be addressed since it is more in line with the actual situation. Furthermore, the vegetation effect on the development of Kelvin-Helmholtz type coherent eddies has never been presented, which plays an important role in the exchange process between the cavity and the main channel.

The present paper focuses on the vortex dynamics of the mixing layer in straight open channels with consecutive vegetated groyne fields (third kind, no sediments). Taking account of the fact that the growth level of plants has a strong seasonal dependence (with a population density that is high in summer and low in winter), this study considers consecutive vegetated cavities with varying population densities of plants, using both laboratory experiments and the large-eddy simulation (LES).

This paper is organized as follows. First, the experimental approach using a two-dimensional particle image velocimetry (PIV) technique is introduced in Sec. II. Next, the LES setup and the model validation are presented in Sec. III. Subsequently, the mean flow patterns inside the embayment, the exchange coefficient across a newly proposed exchange layer, the coherent shedding eddies along the interface, the hydrodynamics of the mixing layer, a phenomenological model for the evolution of the coherent structures, and the discussion are presented in Sec. IV. Finally, the conclusions of the study are summarized in Sec. V.

\section{EXPERIMENTAL APPROACH}

Laboratory experiments are performed in the State Key Laboratory of Water Resources and Hydropower Engineering Science at Wuhan University. The overall experimental system is shown in Fig. 1. The open flume of the rectangular cross section is $20 \mathrm{~m}$ long, $1 \mathrm{~m}$ wide, and $0.5 \mathrm{~m}$ deep, with a $0.1 \%$ bed slope. The inflow with clean water comes from a self-circulation system, which is composed of pipelines, the underground storage, the pump room, and the high pool. As the recirculating water is stored in the underground water tank, the water temperature is relatively stable (about $25^{\circ} \mathrm{C}$ ) over the course of the measurement. The discharge of the inflow is controlled by an electromagnetic flow meter with a precision of $0.1 \mathrm{dm}^{3}$. Honeycomb tubes are installed at the inlet of the (a)

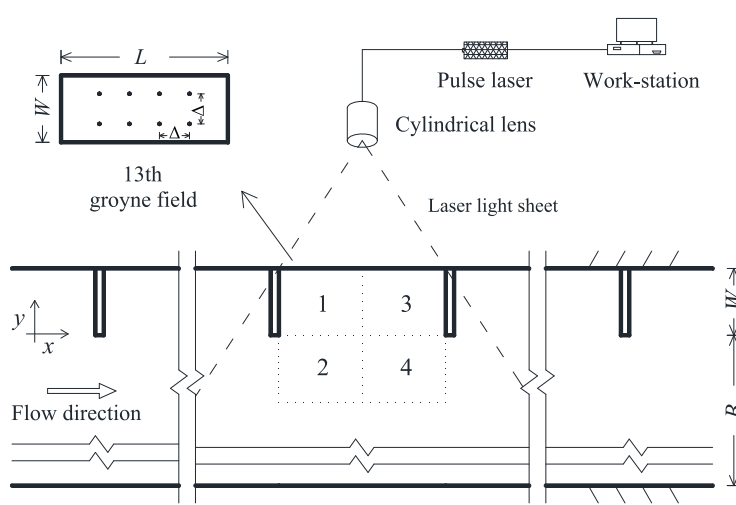

(b)

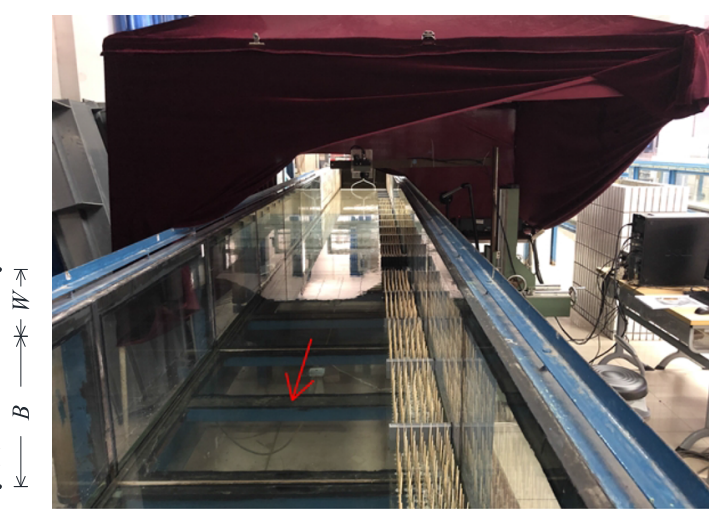

FIG. 1. Overall experimental system: (a) sketch in the horizontal plane and (b) a physical model. 
flume in order to stabilize and straighten the inflow. ${ }^{13}$ A tailgate is located at the end of the flume to adjust the water level. Along the left side of the flume (following the flow direction), 23 groyne models, represented by rectangular elements with sharp corners and hydraulically smooth surfaces $(0.025 \mathrm{~m}$ in the streamwise direction, $0.2 \mathrm{~m}$ in the spanwise direction, and $0.12 \mathrm{~m}$ in the vertical direction), are equidistantly placed to create consecutive groyne fields. These side-cavities are $0.5 \mathrm{~m}$ long in the streamwise direction and $0.2 \mathrm{~m}$ wide in the lateral direction (i.e., $L=0.5 \mathrm{~m}$ and $W=0.2 \mathrm{~m}$, with a fixed aspect ratio $\kappa=0.4$ ), and the bottom of each cavity is in the plane same as that of the main channel. Assuming geometric similarity, straight bamboo sticks, each of diameter $2 \mathrm{~mm}$ and length $12 \mathrm{~cm}$, are arranged regularly in the groyne fields to approximate the rigid emerging vegetation (e.g., reed stems), in an approach similar to that of Xiang et al. ${ }^{18}$ and Sukhodolov et al. ${ }^{38}$ By adjusting the distance $\Delta$ between sticks, it is possible to control the variation in population density of plants. The vegetation density $a$ is defined as follows: ${ }^{48}$

$$
a=\sum_{i=1}^{n} \frac{A_{i}}{S h},
$$

where $A_{i}$ is the frontal area of a single vegetation element of height $h, S$ is the reference bed area of the side-cavity, and $n$ is the number of elements in the groyne field. A right-handed Cartesian coordinate system is adopted, and the origin is set at the tip of the 13th groyne (see Fig. 1). In this coordinate system, the streamwise velocity $u$ is along the $x$-axis, the spanwise velocity $v$ is along the $y$-axis, and the vertical velocity $w$ is along the $z$-axis that originates from the riverbed and is directed toward the free surface.

Based on the results of previous studies, ${ }^{11,14,49,50}$ the inlet discharge $Q$ and the water depth $H$ are fixed to $0.0126 \mathrm{~m}^{3} / \mathrm{s}$ and $0.08 \mathrm{~m}$, and the bulk mean velocity $U=0.197 \mathrm{~m} / \mathrm{s}$ is constant in the mainstream. The Reynolds number $R e \equiv U R / v=13000$ and the Froude number $F r \equiv U / \sqrt{g H}=0.22$. Under these hydraulic conditions, the fluctuations of the free surface in the measurement region are too small to influence the horizontal PIV measurements. As the high vegetation density would shield the laser light sheet noticeably and, thus, strongly affect the measurement accuracy, the experimental tests only include low vegetation densities (see Table I), with no vegetation (case 1), with $\Delta=30 \mathrm{~mm}$ (case 2), and with $\Delta=25 \mathrm{~mm}$ (case 3).

This study chooses the 13th groyne field and its adjacent mainstream area as the measurement region, i.e., parts 1-4 in Fig. 1. The distance between the measurement field and the inflow section is about $8 \mathrm{~m}$, which is nearly 100 times greater than the water depth, ensuring totally developed turbulent flow around the side-cavity. ${ }^{1 .}$ Based on the results of Uijttewaal et al., ${ }^{15}$ McCoy et al., ${ }^{16}$ and

TABLE I. Characteristics of experimental cases.

\begin{tabular}{lccccccc}
\hline \hline Case & $\Delta(\mathrm{mm})$ & $a\left(\mathrm{~m}^{-1}\right)$ & $W(\mathrm{~m})$ & $L(\mathrm{~m})$ & $H(\mathrm{~m})$ & $U(\mathrm{~m} / \mathrm{s})$ & $R e$ \\
\hline 1 & $\ldots$ & 0 & 0.2 & 0.5 & 0.08 & 0.197 & 13000 \\
2 & 30 & 1.92 & & & & & \\
3 & 25 & 2.66 & & & & & \\
\hline \hline
\end{tabular}

Weibrecht et al., ${ }^{32}$ the flow becomes practically periodic (over one cavity length) after the first four to five cavities. The PIV technique is adopted to measure the time-resolved velocity field in the horizontal plane, collecting the $x$-direction velocity $u$ and $y$-direction velocity $v$. The plane at $z=0.05 \mathrm{~m}$ is chosen as the representative measurement elevation to study the flow hydrodynamics around the side-cavity. ${ }^{14}$ A dual-chamber pulse laser (model YAG200-NWL) coupled with a cylindrical lens is employed to generate a $2 \mathrm{~mm}$ thick horizontal pulsed laser light sheet, which shines into the side-cavity from the side of the glass flume. A high-speed charged-coupled device (CCD) camera is located above the cavity to record the particle motion for over 1000 double-frame images of $1600 \times 1192$ pixels with a spatial resolution of $0.18 \mathrm{~mm}$ per pixel, and the time interval between each snapshot is $200 \mathrm{~ns}$. According to previous research, the vortex shedding frequency is dependent on the channel Reynolds number ${ }^{19}$ and it never exceeds $1 \mathrm{~Hz}$ in the range of $R e$ from 8000 to $83000,{ }^{18,28,29,51}$ and then the sampling frequency of $5 \mathrm{~Hz}$ is adopted in this experiment with $R e=13000$, which is high enough to detect the turbulent feature of interest.

The time scale $\Delta t$ between pulses ranges from $1100 \mu$ s to $4500 \mu \mathrm{s}$ (i.e., the laser $\mathrm{A}$ is emitted at $t_{1}=t_{0}$ and the laser $\mathrm{B}$ is emitted at $t_{2}$ $=t_{0}+\Delta t$ ), where the short $\Delta t$ is set for the high-velocity flow in the main channel and the long $\Delta t$ is for the low-velocity flow in the cavity. In the commercial software Insight $4 \mathrm{G}$, double-frame particle images are processed to obtain the time variation of the instantaneous velocity fields. To avoid the wall-attached areas where spurious vectors exist, the processing mask is designed to remove the zones near groynes, and the vector local validation module with the ability to delete the singularity is adopted for the area around straight bamboo sticks. The background subtraction function is performed for original frames in the pre-processing in order to output images that are better suited for vector calculation. ${ }^{50}$ The PIV-algorithm is based on a standard cross correlation via fast Fourier transformation between two images. ${ }^{50}$ The adaptive multi-pass algorithm is adopted, through which the PIV field of view is initially divided into $16 \times 16$ pixels interrogation windows and then further subdivided into $8 \times 8$ pixel interrogation windows with $50 \%$ overlap in each direction. ${ }^{32,50,51}$ Vector local validation and vector conditioning modules are adopted to filter out the spurious vectors and fill the removed vectors through interpolation, respectively. ${ }^{30,31}$ Since the range of each measurement domain is only about $29 \times 22 \mathrm{~cm}^{2}$, four adjacent time-averaged flow fields (parts 1-4 in Fig. 1) at the same elevation are spliced together to form a large flow region of 50 $\times 40 \mathrm{~cm}^{2} .{ }^{13}$ Hollow glass beads, with the diameter ranging from $10 \mu \mathrm{m}$ to $20 \mu \mathrm{m}$ and density $1.05 \mathrm{~g} / \mathrm{cm}^{3}$, are chosen as the tracer particles, which show excellent flow properties.

The Peak Ratio (PR) uncertainty method in Insight $4 \mathrm{G}$ is used to estimate the uncertainty of PIV measurements. The PR method incorporates many possible sources of error (e.g., particle seeding density and pixel displacement), which assumes that errors in pixel displacement follow a Gaussian distribution. According to the uncertainty analysis of the mean measured velocities from multiple samples, ${ }^{51}$ the uncertainty of PIV measurements is $\pm 5 \%$ in the present research. It should be noted that, in the preliminary experiment, seven points [points a to $\mathrm{g}$ in Fig. 2(a)] at $z=0.05 \mathrm{~m}$ in case 1 are chosen as survey locations where the time-averaged velocity $\bar{u}_{P I V}$ measured from PIV is compared with that from a portable flow meter (PFM). Figure 2(b) shows the comparison of time-averaged 
(a)

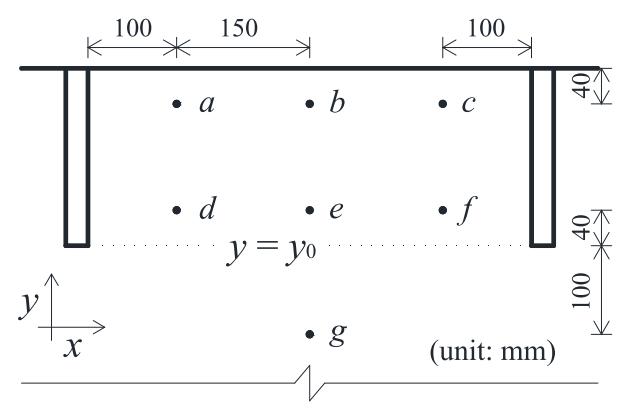

(b)

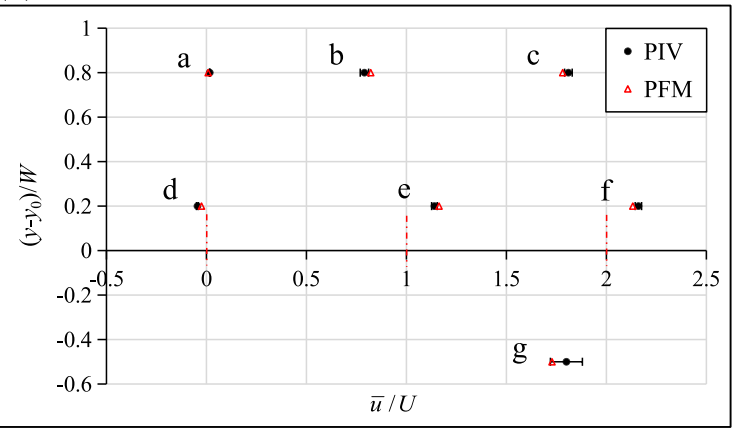

FIG. 2. Comparisons of the time-averaged streamwise velocity at $z=0.625 \mathrm{H}$ between PIV and PFM: (a) sketch of the selected positions; (b) case 1.

streamwise velocities between PIV and PFM, together with the $10 \%$ deviation of $\bar{u}_{P I V}$, which exhibits a good consistency.

\section{NUMERICAL SIMULATION}

As the laboratory experiment only studies the cases with low vegetation densities, we additionally perform the LES to investigate the effect of high vegetation densities $[\Delta=20 \mathrm{~mm}$ (case 4) and $\Delta=17 \mathrm{~mm}$ (case 5), Table II] in the groyne field, together with low vegetation density cases (case 1-3) for validations. The details of LES cases are summarized in Table II.

\section{A. Model equations}

Spatial filtering of the incompressible Navier-Stokes equations gives the governing equations for the LES, i.e., the continuity and momentum equations, as follows:

$$
\begin{gathered}
\frac{\partial \tilde{u}_{i}}{\partial x_{i}}=0 \quad(i=1,2,3), \\
\frac{\partial \tilde{u}_{i}}{\partial t}+\frac{\partial}{\partial x_{j}}\left(\tilde{u}_{i} \tilde{u}_{j}\right)=-\frac{1}{\rho} \frac{\partial \tilde{p}}{\partial x_{i}}+\frac{\partial}{\partial x_{j}}\left[v\left(2 \tilde{S}_{i j}\right)-\tau_{i j}\right],
\end{gathered}
$$

where the overbar represents resolved variables, $u_{i}(\mathrm{~m} / \mathrm{s})$ is the velocity component in direction $i, \rho\left(\mathrm{kg} / \mathrm{m}^{3}\right)$ is the density, $p\left(\mathrm{~N} / \mathrm{m}^{2}\right)$ is the dynamic pressure, $v\left(\mathrm{~m}^{2} / \mathrm{s}\right)$ is the kinematic viscosity, and $S_{i j}$ $=\frac{1}{2}\left(\partial u_{i} / \partial x_{j}+\partial u_{j} / \partial x_{i}\right)\left(\mathrm{s}^{-1}\right)$ is the strain-rate tensor. $\tau_{i j}=\widetilde{u_{i} u_{j}}-\tilde{u}_{i} \tilde{u}_{j}$ $\left(\mathrm{m}^{2} / \mathrm{s}^{2}\right)$ is the subgrid-scale (SGS) stress, representing the effect of the unresolved small-scale motions on the resolved flow field, which is determined from the following eddy-viscosity model:

$$
\tau_{i j}-\frac{1}{3} \delta_{i j} \tau_{k k}=-v_{t}\left(2 \tilde{S}_{i j}\right),
$$

where $v_{t}\left(\mathrm{~m}^{2} / \mathrm{s}\right)$ is the eddy viscosity. In this study, the dynamic Smagorinsky model proposed by Germano et al. ${ }^{52}$ is employed as the SGS model to calculate $v_{t}$, thus avoiding the problems of the earliest SGS model, in which dissipation was over-predicted and the inverse energy cascade was not taken into account.

\section{B. Simulation setup}

The commercial software FLUENT (version 14.5) is used to perform the LES in this study. The finite volume method (FVM) is utilized to discretize the governing equations, and the pressurevelocity coupling scheme is implemented by the SIMPLE method. Bounded central differencing for spatial discretization is used to solve the convection-diffusion equations, and a bounded secondorder implicit method is used for time-stepping. ${ }^{54}$ The solution is iterated many times within a time step, and it is considered to be convergent when the residual is smaller than $1 \times 10^{-4}$.

The flow region around the 13th groyne field is taken as the computational domain (see Fig. 3). A right-handed Cartesian coordinate system is adopted here, the same as that in the experiment. As mentioned in Sec. II, the flow could be regarded as periodic when it arrives at the computational domain, and therefore, the periodic condition is used at both the inlet and outlet. ${ }^{11,12,55}$ According to

TABLE II. Summary of numerical simulation cases.

\begin{tabular}{ccccccccc}
\hline \hline Case & $\Delta(\mathrm{mm})$ & $a\left(\mathrm{~m}^{-1}\right)$ & Time step $(H / U)$ & Grid number $\left(\times 10^{6}\right)$ & $W(\mathrm{~m})$ & $L(\mathrm{~m})$ & $H(\mathrm{~m})$ & $U(\mathrm{~m} / \mathrm{s})$ \\
\hline 1 & $\ldots$ & 0 & 0.00625 & 19 & & & \\
2 & 30 & 1.92 & 0.005 & 25 & 0.2 & 0.5 & 0.08 & 0.197 \\
3 & 25 & 2.66 & 0.005 & 30 & & & \\
4 & 20 & 4.32 & 0.005 & 36 & & \\
5 & 17 & 6.16 & 0.005 & 38 & & \\
\hline \hline
\end{tabular}


(a)

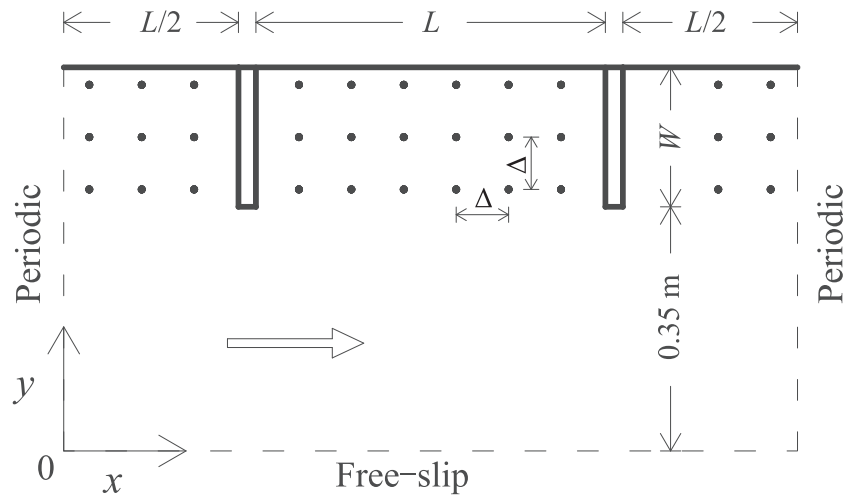

(b)

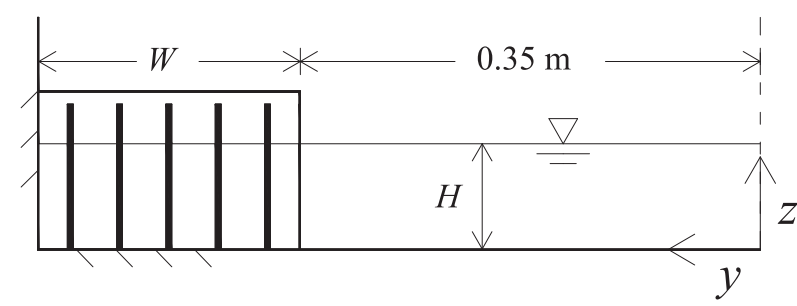

FIG. 3. Schematic diagram of the simulation domain: (a) view in the horizontal plane and (b) sketch of the cross section.

Alfrink and Van Rijn, ${ }^{56}$ the rigid-lid approximation for the free surface of an open-channel flow is generally acceptable for $\mathrm{Fr}<0.5$. In this study with $F r=0.22$, the free surface can be treated as a rigid lid. To reduce the computational resources, we only select $0.35 \mathrm{~m}$, i.e., $1.75 \mathrm{~W}$, as the simulated width of the main-stream, which can guarantee that the right boundary (along the flow direction) of the simulated domain could not be affected by the cavity. ${ }^{11}$ A free-slip condition is imposed at the right boundary, and no-slip conditions are imposed on the walls and the surfaces of the vegetation.

For all the simulated cases, structured hexahedral grids are employed to discretize the whole computational domain, and the $\mathrm{O}$-grid block method is used to model the rigid vegetation. Because the mesh distributions in different cases are similar, only the grid in case $4(\Delta=20 \mathrm{~mm})$ is described here in detail, as shown in Fig. 4 . For the LES technique, the mesh around the vegetation and walls must be sufficiently fine to avoid any use of wall functions, i.e., the near-wall region must be resolved. ${ }^{57}$ In our LES, the grid size near the surface of the solid wall is close to one wall unit, i.e., $y^{+}=1$. At positions away from the wall, the maximum grid size reaches 15 , 19 , and 19 wall units for the $x$-, $y$-, and $z$-directions, respectively. Totally, approximately $36 \times 10^{6}$ nonuniform structured grids are generated in case 4 with a time step of $0.005 \mathrm{H} / \mathrm{U}$. The grid information for the other cases is summarized in Table II. For all the cases, simulations are first running for 24 flow-through times until the transients are eliminated, where one flow-through time is equal to $L / U$, i.e., the time taken for the water to flow through one groyne field length. ${ }^{11,12}$ Then, instantaneous flow fields are collected for a time period of $32 \mathrm{~L} / \mathrm{U}$, which is actually sufficient for the turbulent statistic to achieve stationary statistical profiles. ${ }^{16}$

\section{Model validation}

Figure 5 compares the results of the time-averaged streamwise velocity $\bar{u}$ (normalized by $U$ ) at $z=0.625 H$ obtained from the PIV and LES for cases 1 and 2. For both cases, the numerical results show a good consistency with the experimental data, which demonstrates the reliability of the LES. It should be noted that, in the regions marked by the dashed red ovals, the flow velocity from the LES is slightly larger than that from the laboratory experiments (the maximum relative error is about 7\%). The velocity offset might come from the inlet condition. In our LES, the inlet flow rate is prescribed by multiplying the simulated width by the bulk mean (a)

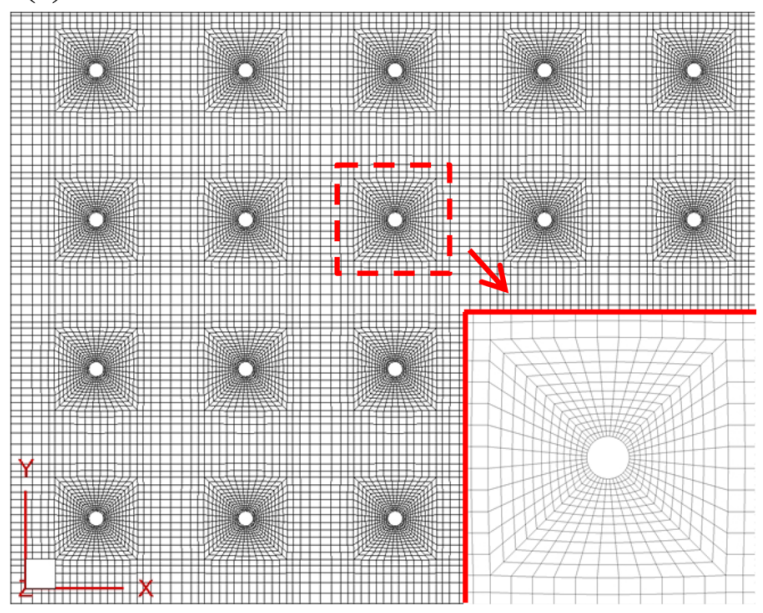

(b)

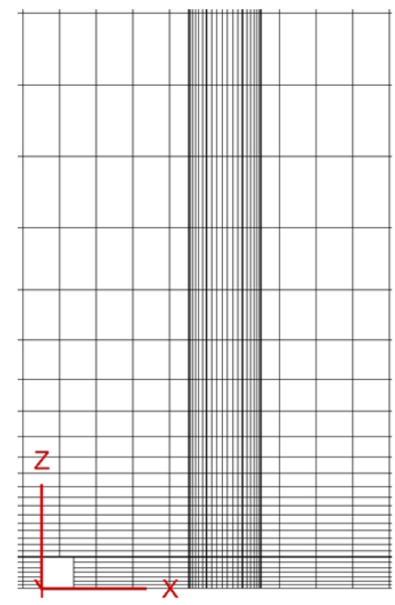

FIG. 4. Computational mesh in case 4: (a) mesh around the vegetation; (b) mesh near the wall. 
(a)

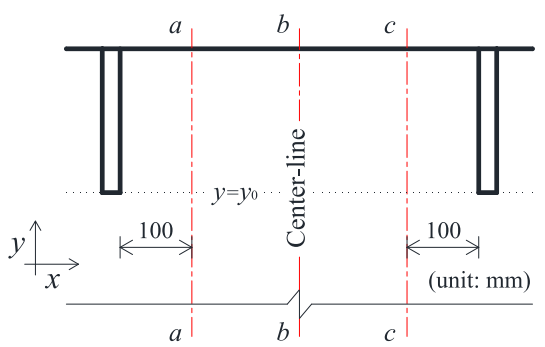

(b)

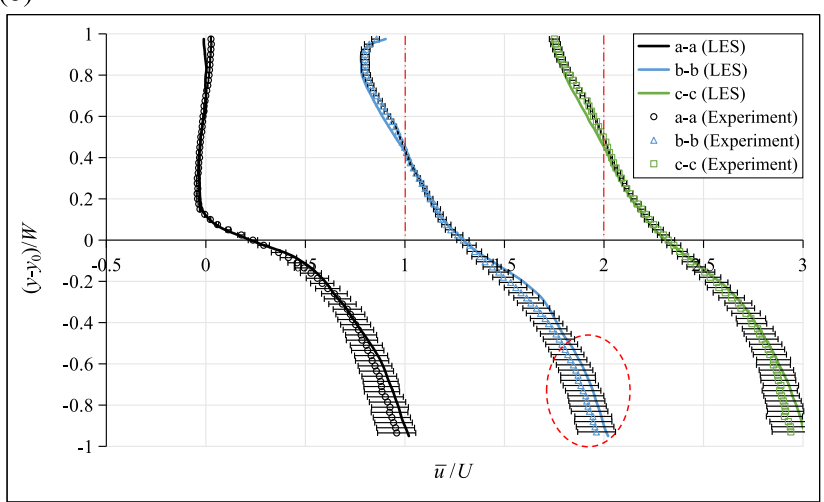

(c)

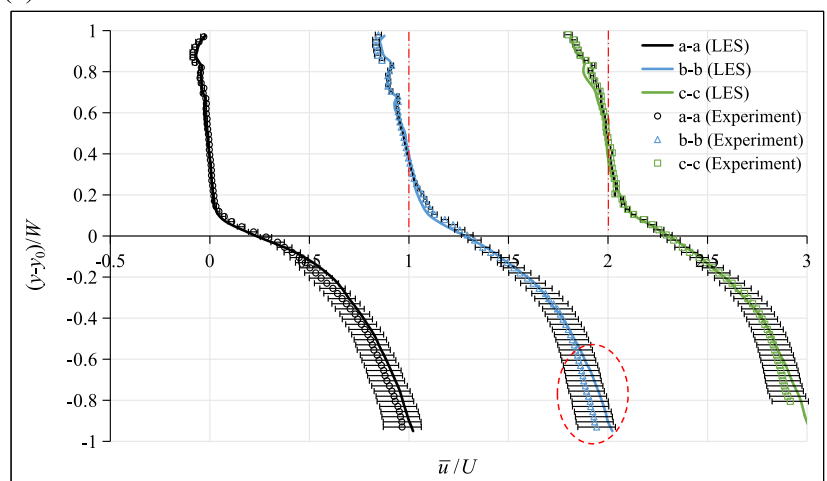

FIG. 5. Comparisons of the time-averaged streamwise velocity at $z=0.625 \mathrm{H}$ between the PIV and LES: (a) sketch of the selected positions; (b) case 1; and (c) case 2 .

velocity $U,{ }^{11,12}$ thus magnifying the low flow velocity near the groyne field, which causes the inlet flow rate in the LES to be slightly larger than that in the laboratory experiment and eventually leads to the velocity differences in the main-stream. When $\left(y-y_{0}\right) / W$ is larger than approximately 0.4 , the streamwise velocity becomes negative, which indicates that a recirculating flow has existed in the groyne field. Note that the velocity distribution in case 2 is fluctuating at $(y$ $\left.-y_{0}\right) / W=0.84$, which is caused by the Kármán vortex street generated by the vegetation. The validations of cases 1 and 2 indicate that the present LES is reasonable in modeling this kind of flow.

\section{RESULTS AND DISCUSSION}

\section{A. Flow pattern}

Figure 6 shows the mean velocity vectors $(\bar{u}, \bar{v})$ obtained from PIV in the horizontal plane of $z=0.625 H$. When the vegetation density in the side-cavity increases, the double circulation system (i.e., a large primary circulation accompanied by a small secondary circulation) in case 1 develops into a single circulation structure in cases 2 and 3, which is consistent with the observations of Sukhodolov et al. ${ }^{38}$ The similarity of flow patterns between these two kinds (the second and third kinds) might be attributed to the fact that the flow structure inside the groyne field is strongly affected by the vegetation patches, and the effect of upstream and downstream vegetated cavities is small compared with the vegetation patches inside the groyne field. This paper considers that the vegetation itself and its drag force are the essential reasons for the transformation of the circulation structure. First, the vegetation can block part of the high-velocity jet-like flow attached to the downstream groyne into the side-cavity, and the jet-like flow is regarded as the main driving force for the primary circulation. ${ }^{16}$ Second, the drag force of the vegetation in the inner embayment further reduces the momentum of the primary circulation that is assumed to be the driving force for the secondary circulation. ${ }^{58}$ As the combined result of these two effects, the primary circulation velocity in vegetated cases is too small to generate a second gyre.

Figure 7 shows the stream-lines of the time-averaged flow field from the LES at $z=0.625 \mathrm{H}$. It is clear that different vegetation densities can change the circulation system in the groyne field. In Figs. 7(a), i.e., in the absence of vegetation, two gyres are observed in the side-cavity, similar to Fig. 6(a). When the amount of vegetation increases to the level of case 2 , the space occupied by the secondary gyre is clearly reduced [see Fig. 7(b)]. With a further increase in the vegetation density, the secondary circulation disappears completely [see Figs. $7(\mathrm{c})-7(\mathrm{e})$ ]. Note that as the flow pattern changes, the center of the primary gyre moves correspondingly. The center of the rotating stream-lines, marked by the red cross-hairs in Fig. 7, is defined as the core of the circulation structure, referring to Jackson et al. ${ }^{51}$ It moves from the position $(0.67 \mathrm{~L}, 0.44 \mathrm{~W})$ in case 1 (taking the tip of the upstream groyne as the origin), to $(0.75 \mathrm{~L}, 0.40 \mathrm{~W}),(0.84 \mathrm{~L}$, $0.30 W),(0.84 L, 0.31 W)$, and $(0.87 L, 0.26 W)$ in cases $2-5$, respectively. The tendency of movement is downstream in the $x$-direction and toward the interface in the $y$-direction.

To quantitatively assess the effect of vegetation on the circulation structure, the distributions of mean streamwise velocity along the $y$-direction at $z=0.625 \mathrm{H}$ are shown in Fig. 8. The vegetation clearly reduces the circulation velocity in the groyne field, and the reduction effect is enhanced with an increase in the vegetation densities. The mean streamwise velocity in the primary gyre of case 1 is about $19 \%$ of that in the main-stream. The spatial averaged value is calculated as $\sum_{i=1}^{n}\left|\bar{u}_{\left(x_{i}, y_{i}\right)}\right| / n$, where $\bar{u}_{\left(x_{i}, y_{i}\right)}$ represents the time-averaged velocity at different locations with the elevation of $z$ $=0.625 \mathrm{H}$ in the primary gyre. In case 3 , the circulation velocity has dropped to $0.13 U$ owing to the presence of vegetation. When the distance $\Delta$ between plants further decreases to $17 \mathrm{~mm}$ (case 5), the circulation velocity reaches its minimum, $0.08 U$.

Figure 9 shows stream-lines of the mean flow at different cross sections, where color-contours represent the resultant velocity of 
(a)

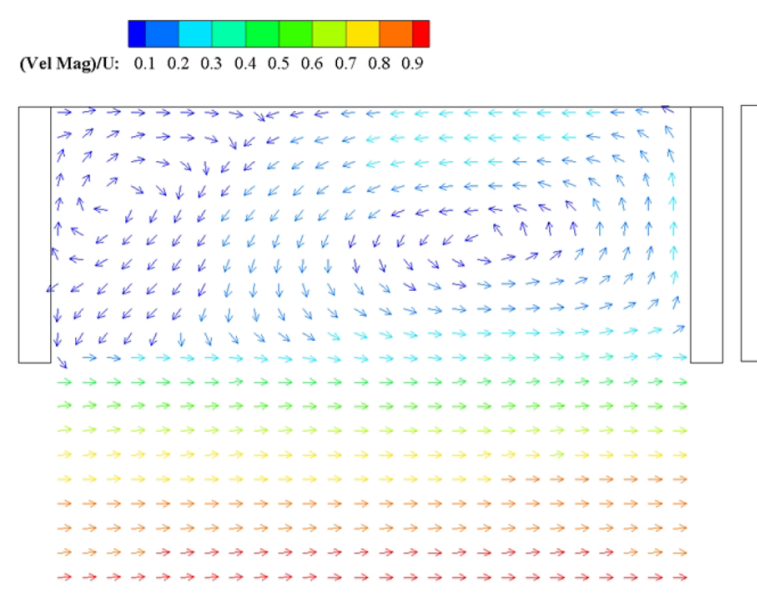

(b)

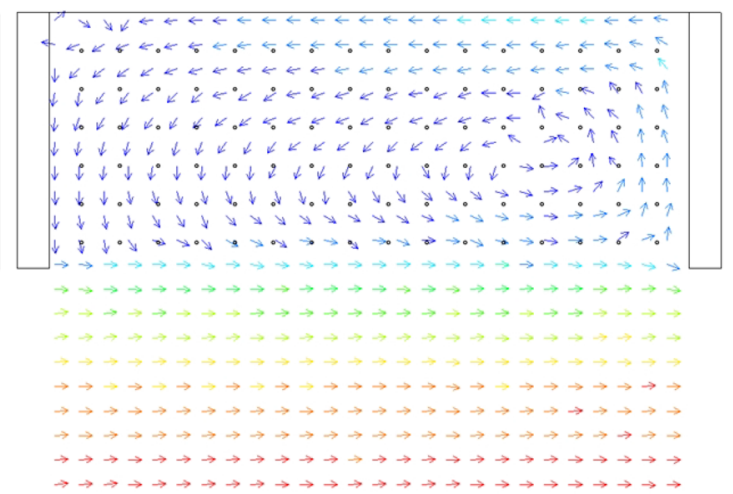

(c)

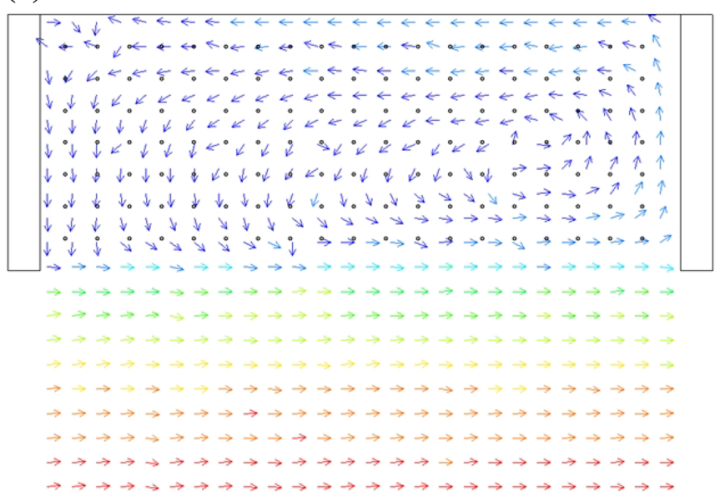

FIG. 6. Time-averaged two-dimensional velocity vectors at $z=0.625 \mathrm{H}$ from PIV: (a) case 1; (b) case 2; and (c) case 3 .

$\bar{v}$ and $\bar{w}$, normalized by $U$. For all the cases, a pair of streamwise secondary currents (i.e., the top secondary current and the bottom one) is formed in the main-stream region immediately adjacent to the cavity. These two secondary flows occupy nearly half the water depth, and they are not vertically symmetric, with cores close to the interface. This streamwise double-vortex system could lead to a helicoidal current when superimposed to the main-stream, thus affecting the dynamic of sediment transport in this zone, as described by Brevis et al. ${ }^{11}$

As for the secondary current in the groyne field, it barely exists in the non-vegetation case, and stream-lines here are almost parallel to the $y$-axis, probably due to the strong advective current between the cavity and the main channel. With the increase in the vegetation density, the advective motion is suppressed by plants, and the secondary current occurs inside the side-cavity, particularly evident in case 5 . However, the velocity magnitude of the internal secondary current is too small to generate an effective flow in the $z$-direction. Therefore, the recirculating flow in the cavity can be regarded as a horizontal two-dimensional motion.

\section{B. Exchange coefficient}

Mass exchange between the groyne field and the main channel plays an important role in the eco-environment of a river. On the one hand, transfer of nutrients from the main-stream to the embayment facilitates the growth of aquatic organisms in the side-cavity, which can enhance local biodiversity. On the other hand, contaminants (e.g., polluted sediment) accumulating inside the cavity can act as a source of pollution under flood conditions. ${ }^{59}$

Based on the relation between hydrodynamics and water quality, it is confirmed that mass exchange across the interface is essentially determined by the corresponding momentum exchange. Figure 10 shows the mean velocity $\bar{v}$ (normalized by $U$ ) from the LES in different vertical slices near the interface. In cases with low vegetation density (cases 1-3), the flow enters the cavity mainly from the downstream part of the interface, together with a few coming from the bottom layer of the interface. The flow escapes from the cavity through the upstream part of the interface. Note that as the vegetation density increases from case 1 to case 3 , the inflow range and 
(a)

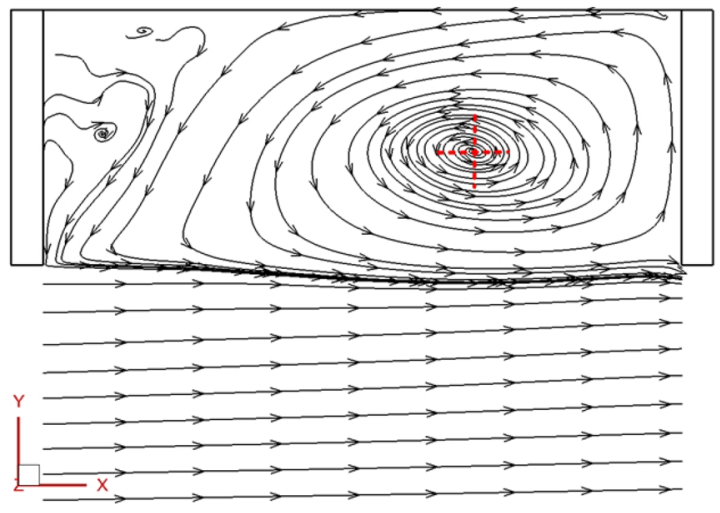

(c)

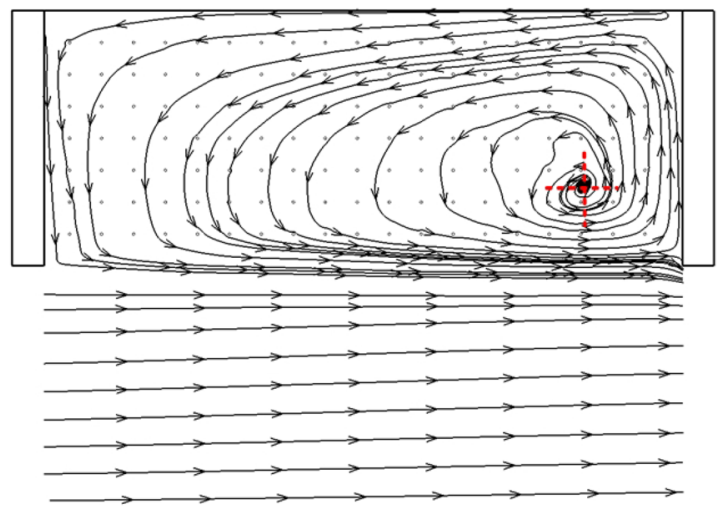

(b)

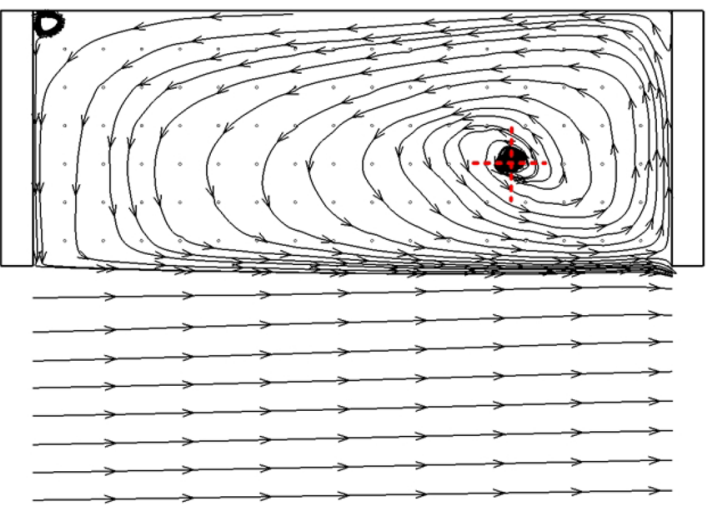

(d)

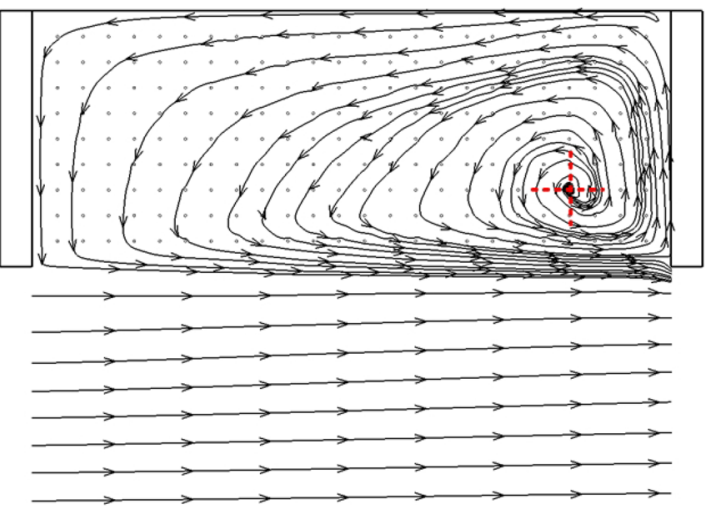

(e)

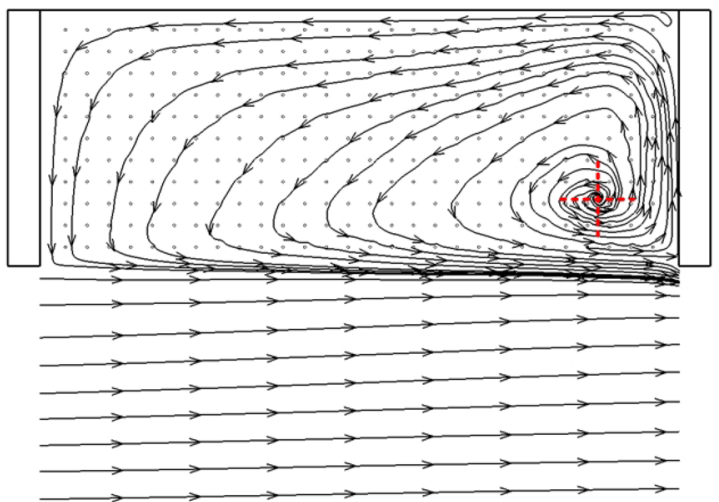

FIG. 7. Time-averaged two-dimensional stream-lines at $z=0.625 \mathrm{H}$ from the LES: (a) case 1 ; (b) case 2; (c) case 3; (d) case 4; and (e) case 5 .

the entrance velocity in the downstream part gradually decrease as a consequence of the blocking effect of the vegetation, as discussed in Sec. IV A. In cases with a larger vegetation density (cases 4 and 5), the inflow across the full depth observed in cases 1-3 is no longer present because the vegetation weakens the inflow to some degree that it is unable to form a complete wall-attached jet-like flow. For cases 4 and 5, the inflow toward the groyne field is primarily located at the bottom layer of the interface, with the outflow toward the main channel almost occupying the middle and top layers of the interface beside the position of the free surface where the inflow occurs. 
(a)

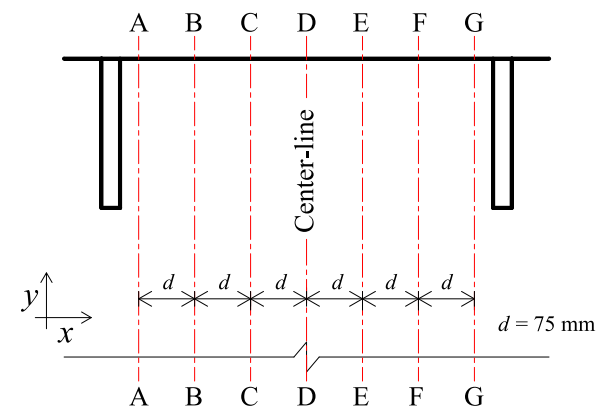

(b)

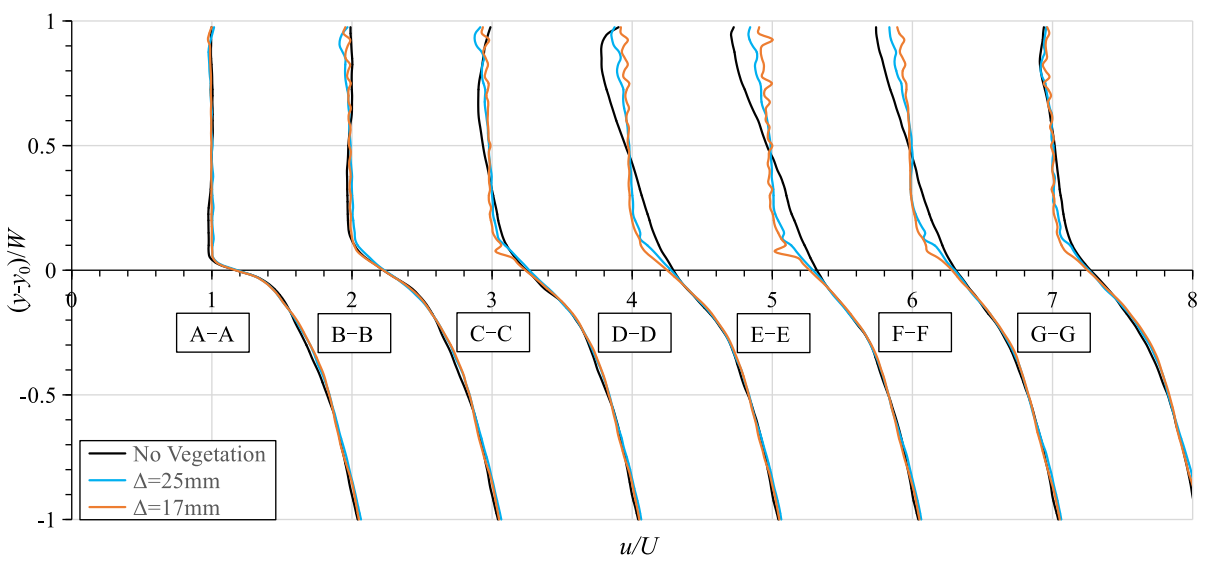

FIG. 8. Mean streamwise velocity along the $y$-direction at $z=0.625 \mathrm{H}$ from the LES: (a) sketch of the selected positions and (b) mean streamwise velocity distribution for different cases.

Through the momentum exchange process, materials such as nutrients dissolved in the water are transferred between the cavity and the main channel. To provide a quantitative evaluation of the exchange efficiency across the interface, the concept of exchange coefficient $K\left(\mathrm{~s}^{-1}\right)$ is defined as follows: $:^{15,27,29,49,58}$

$$
K=\frac{H_{i} \bar{E}}{H_{e} W}
$$

where $H_{i}(\mathrm{~m})$ is the water depth at the interface and $H_{e}(\mathrm{~m})$ is the mean depth in the embayment. $\bar{E}(\mathrm{~m} / \mathrm{s})$ is the mean exchange velocity across the interface, which can be obtained by averaging the instantaneous exchange velocity $E$ defined in Eq. (7). In this paper, $H_{i}$ and $H_{e}$ are of the same value, equal to the water depth $H$ of the main-stream. Usually, $K$ is normalized by the main-stream velocity $U$ and the embayment width $W$, in the combination of $U / W$, and thus, the new dimensionless exchange coefficient $k$ is defined as follows: ${ }^{60}$

$$
k=\frac{K}{U / W}=\frac{H_{i} \bar{E}}{H_{e} U}=\frac{\bar{E}}{U} .
$$

According to the formula proposed by Weitbrecht and Jirka, ${ }^{61}$ the instantaneous exchange velocity $E(\mathrm{~m} / \mathrm{s})$ is calculated as

$$
E=\frac{1}{2 A_{i}} \int_{A_{i}}|v|
$$

where $A_{i}\left(\mathrm{~m}^{2}\right)$ is the area of the interface and $v(\mathrm{~m} / \mathrm{s})$ is the transverse velocity of an infinitesimal element $d A$ of the interface.

At this point, the dimensionless exchange coefficient $k$ could be found straightforwardly from the above-mentioned procedure. However, a crucial question arises as to whether the chosen interface [the first slice in Fig. 10(a)] can represent the true exchange layer. In other words, is the exchange coefficient $k$ calculated from the first slice correct or not? Before answering this question, it is necessary to further analyze the distribution of transverse velocity in Fig. 10. From the flow field near the downstream groyne, an important detail can be observed for all the cases: there is a thin layer of high-velocity outflow adjacent to the groyne in the range from $y=0.35 \mathrm{~m}$ to $y$ $=0.358 \mathrm{~m}$ (as indicated by the green region), and when $y>0.358 \mathrm{~m}$, the inflow toward the cavity (orange) occurs. This phenomenon can be explained through the sketch of the flow, as shown in Fig. 11. As flow A from the main channel initially enters the groyne field, its direction is inclined to the groyne, and then, the flow crashes violently into the groyne. After this crash, flow A separates into two parts: flow $\mathrm{B}$ and flow C. Flow B, i.e., the inflow in the range $y$ $>0.358 \mathrm{~m}$, develops into a wall-attached jet-like flow. Flow C, i.e., the outflow in the range $y<0.358 \mathrm{~m}$, bypasses the tip of the groyne and flows downstream. Thus, flow $\mathrm{C}$ does not actually participate in mass exchange between the groyne field and the main channel, and choosing the interface (i.e., the first slice in Fig. 10) as the exchange layer leads to some error. 
(a)

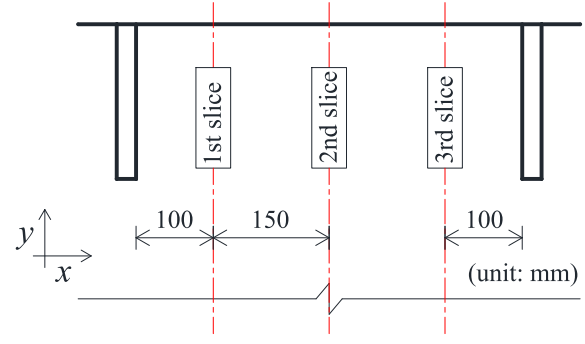

(c)

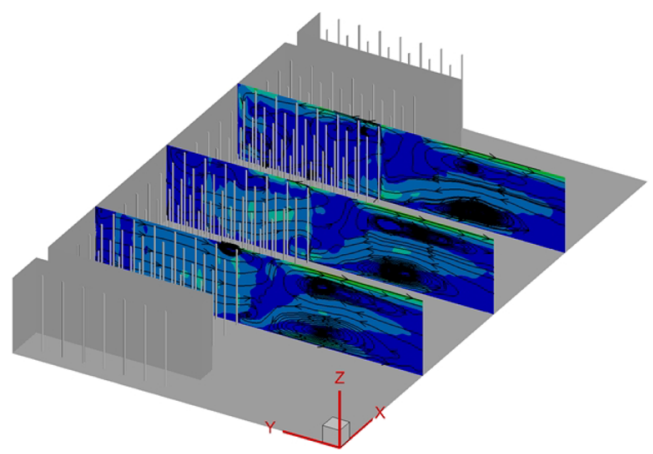

(b)

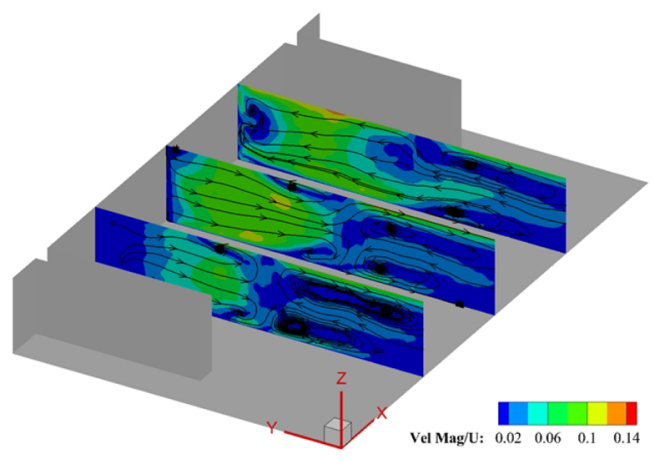

(d)

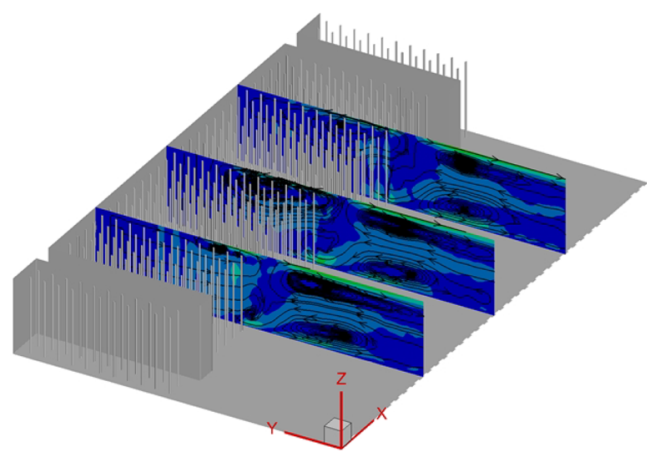

FIG. 9. Time-averaged cross-sectional stream-lines superimposed with the resultant velocity of $\bar{v}$ and $\bar{w}$ from the LES (the ratio of vertical to streamwise scales is 1.15:1): (a) sketch of the selected slices; (b) case 1; (c) case 3; and (d) case 5.

Based on the above-mentioned analysis, the exchange layer is now taken to be the vertical plane with the $y$ coordinate at the crash position in Fig. 11, which prevents flow $\mathrm{C}$ from participating in the exchange process. It can be seen from Fig. 10 that for all the cases, the crash position is located at about $y=0.358 \mathrm{~m}$, i.e., the fifth slice, at a distance $0.04 \mathrm{~W}$ from the tip of the groyne. Figure 12 shows the exchange coefficient $k$ calculated from the LES with the newly chosen exchange layer (the fifth slice) for different vegetation densities. Overall, $k$ gradually decreases with an increase in the vegetation density, and it is considered that the blocking effect of the vegetation is enhanced with higher densities such that it is able to suppress the transverse momentum exchange across the exchange layer, as shown in Fig. 10, thus leading to a decrease in $k$, which is in contrast with Sukhodolov et al., ${ }^{38}$ i.e., vegetation patches inside the cavity have negligible effects on the characteristics of the shear layer at the interface between main flow and groyne field. This difference could be attributed to the fact that the upstream wake effect of the vegetation patches is not taken into account in the second kind configuration (multiple groyne fields with vegetation in a single cavity). Note that from a complete absence of vegetation to sparse vegetation (from case 1 to case 2), there is a fairly sharp drop in the value of $k$ by about $10 \%$, whereas in the vegetated cases, going from case 2 to case 5 , the rate of decrease in $k$ is small, no more than $2 \%$.

\section{Coherent shedding eddy}

Using flow visualization techniques (e.g., PIV) or the LES, previous studies have revealed the eddy shedding phenomenon along the mixing layer. ${ }^{15,19,20,32}$ These eddies are produced behind the tip of the groyne and then moved downstream in a quasiperiodic manner, which exhibit the coherent character. To investigate vegetation effects on the development of such coherent eddies, Figs. 13 and 14 show the eddy development from the laboratory experiments in cases 1 and 2, respectively, where vertical vorticity fields are superimposed with temporal velocity vectors, referring to Sanjou et al. ${ }^{30}$ and Weitbrecht et al. ${ }^{32}$

In the absence of vegetation, a small-scale eddy marked by the black circle in Fig. 13(a) is initially formed behind the tip of the groyne as a result of the large velocity gradient between the cavity and the main channel. As this eddy migrates downstream along the interface, it randomly merges with other eddies that have been shed. During the merging process, surrounding water from the sidecavity is sucked in. As shown in Figs. 13(b)-13(d), when mixing proceeds, the merged eddy grows in size compared with the initial eddy, as in Fig. 13(a). Note that, in Fig. 13(d), as marked by the small black circle, a new eddy has been shed, which will develop in the similar way. This periodic process eventually generates the 
(a)

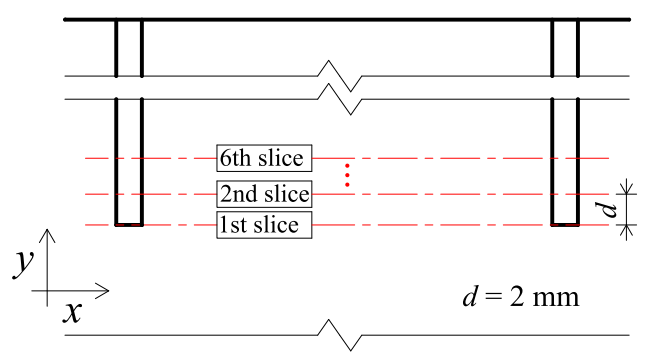

(c)

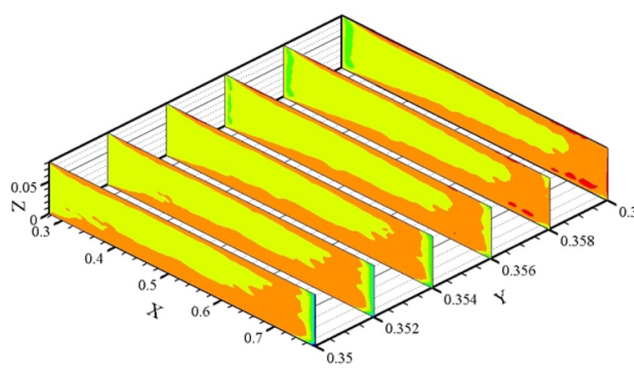

(e)

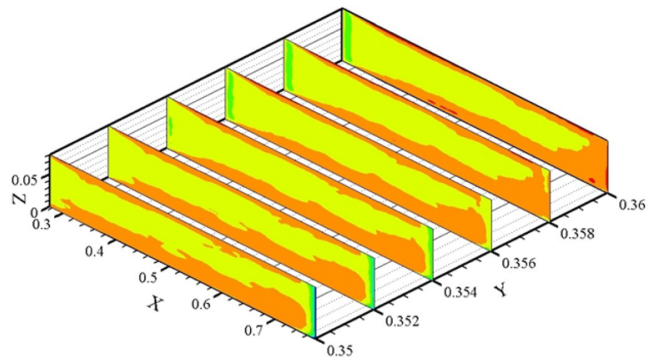

(b)

$\mathbf{v} / \mathbf{U}: \quad-0.25-0.2-0.15-0.1-0.05 \quad 0 \quad 0.05$

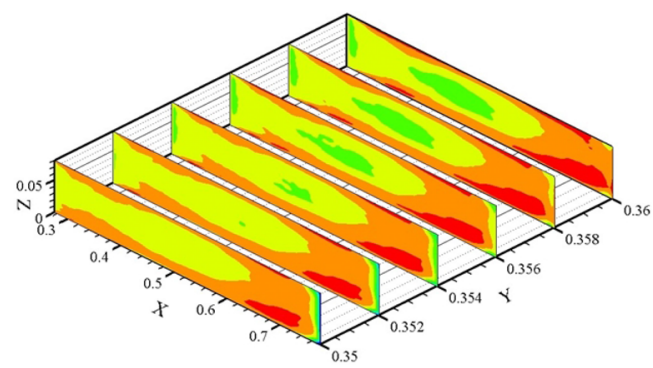

(d)

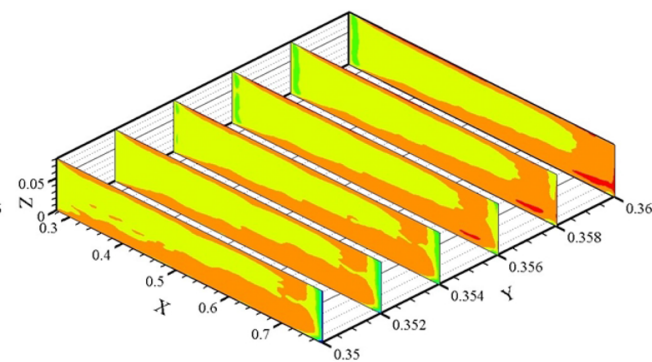

(f)

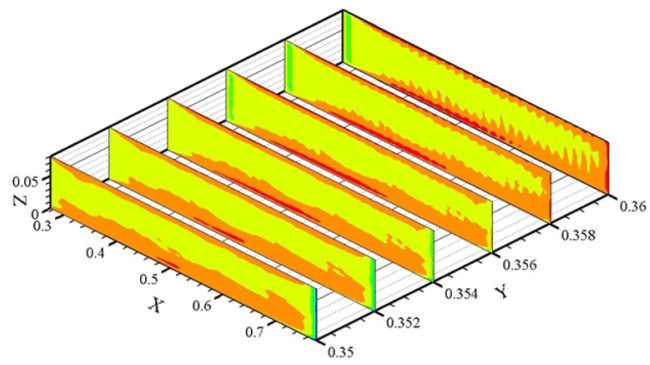

FIG. 10. Time-averaged spanwise velocity from the LES in different slices along the $y$-direction (the ratio of spanwise to streamwise scales is $55: 1$ ): (a) sketch of the selected positions; (b) case 1; (c) case 2; (d) case 3; (e) case 4; and (f) case 5.

large-scale coherent structures in the mixing layer. As in the cases with no vegetation, mergers and the entry of surrounding water can cause the eddy shed in the vegetated case to gradually increases in size when it flows downstream (Fig. 14). However, the scale of the merged eddy in case 2 [Figs. 14(b)-14(d)] is smaller than that in case 1 [Figs. 13(b)-13(d)]. This difference could be induced by the presence of the vegetation in case 2 . On the one hand, the plants act as a constraining boundary to limit the free growth of the eddy. On the other hand, the blocking effect of the plants reduces the amount of surrounding water entering the merging eddy.

To provide a better understanding of these Kelvin-Helmholtz eddies, 3D coherent structures of the vortex system visualized by the $Q$-criterion are shown in Fig. 15. If the value of $|Q|$ is larger in some region, it means that the rate of rotation is stronger there. For all the cases, the coherent structures are illustrated by snapshots of an iso-surface of $Q=55 \mathrm{~s}^{-2}$ colored with the water depth for clarity. Overall, the large-scale coherent structures with high vorticity are concentrated in the mixing layer, where a large velocity gradient and unsteady shearing motion are present. When the vegetation density increases, both the extent of penetration of the coherent vortices into the cavity and the coverage area of Kármán vortices generated behind the plants gradually decrease, which could lead to a decrease in the average turbulent kinetic energy (TKE) in the groyne field.

Referring to the previous studies, ${ }^{16,18,21,29}$ the coherent motions of eddies could be detected in the upstream of the mixing layer where a distinct velocity-gradient exists as the inducing condition for vortex shedding phenomena. Based on the above-mentioned principle 


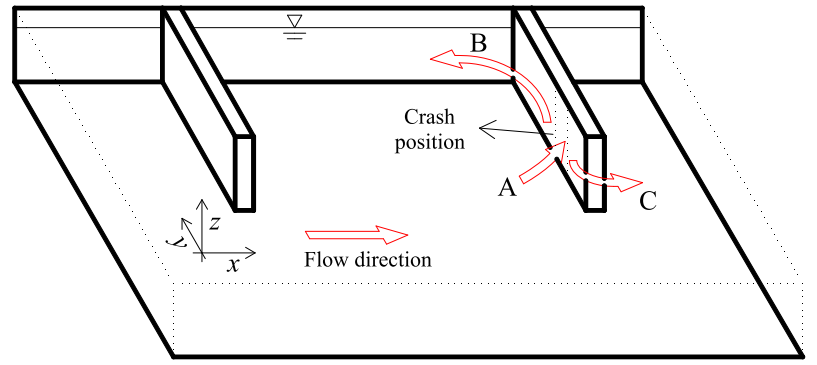

FIG. 11. Sketch of flow near the downstream groyne.

and the visualization result of vortex developments in the experiment (see Figs. 13 and 14), the location of point A [see Fig. 16(a)] is determined as the monitoring location to quantify the dominant time scales of coherent eddies. The characteristic frequencies of coherent motions are obtained from the transverse velocity spectrum at point $\mathrm{A}$ in the LES, as shown in Fig. 16. The power spectra, with the frequency resolutions of $0.0122 \mathrm{~Hz}$ for case 1 and $0.0153 \mathrm{~Hz}$ for other cases, are computed through the Fourier Transformation procedure. The frequency $f$ is normalized as the Strouhal number, $S t=f H / U$. In Fig. 16(b), a -3 subrange appears here, as well as the Kolmogorov $-5 / 3$ inertial subrange typically for 3D turbulent flows. The presence of a -3 subrange indicates a strong energy exchange from small scales to large ones. ${ }^{16}$ This energy exchange can be attributed to the merging of shedding eddies. Further evidence for this mechanism is provided by the partial power spectra in Figs. 16(c)-16(e). In Fig. 16(c), i.e., case 1, the first energy peak is at $S t_{1}=0.117$, corresponding to a frequency $f_{1}=0.29 \mathrm{~Hz}$, which represents the dominant time scale of the merging process, as described by McCoy et al. ${ }^{19}$ The second energy peak is at $S t_{2}=0.200$, corresponding to a frequency $f_{2}=0.50 \mathrm{~Hz}$, which is associated with the shedding of vortices behind the groyne. In Figs. 16(d) and 16(e), i.e., cases 3 and 5, the values of $S t_{1}$ are 0.138 and 0.219 , respectively, corresponding to frequencies $f_{1}$ of $0.35 \mathrm{~Hz}$ and $0.55 \mathrm{~Hz}$, and the values of $S t_{2}$ are 0.366 and 0.396 , respectively, corresponding to frequencies $f_{2}$ of $0.91 \mathrm{~Hz}$ and $0.99 \mathrm{~Hz}$. It is found that with increasing vegetation densities, the leading frequency $f_{1}$ of the merging eddy becomes larger, which is caused by the gradual decrease in the scale of the

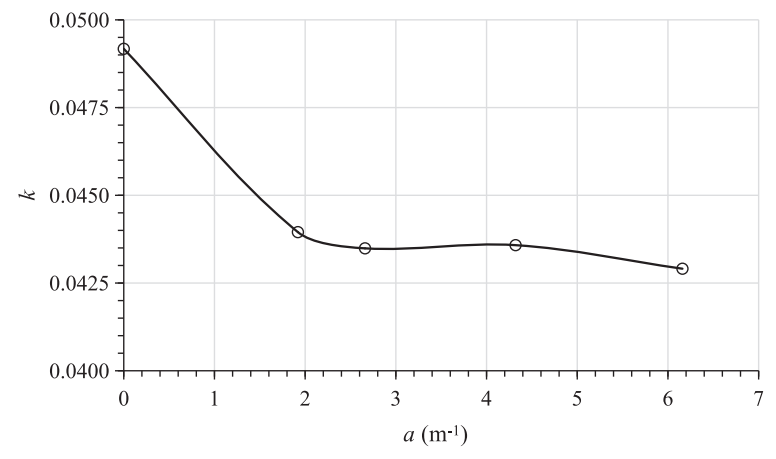

FIG. 12. Variation of the exchange coefficient $k$ with the vegetation density a. merging eddy, as mentioned earlier in this subsection. Previous studies showed that the dominant frequency is negatively correlated with the vortex size. ${ }^{8,19}$ The master frequency $f_{2}$ of eddy shedding increases with increasing vegetation density. This may be due to the fact that the presence of vegetation increases the velocity gradient in the mixing layer, leading to a growth in the instability of the KelvinHelmholtz shear layer ${ }^{47}$ and, thus, to the acceleration of the vortex shedding.

It should be noted that as the coherent motions have been observed around point $\mathrm{A}$ in the experiment (see Figs. 13 and 14), this point is selected as the monitoring location in the LES to quantify dominant time scales of coherent eddies. However, for the situation without the help of visualization techniques, the merging location is hard to identify since the vortex merging in the mixing layer is a highly localized phenomenon. ${ }^{62}$ It is more feasible to plot the spectra from several locations in the streamwise direction. In addition, wavelet analysis is considered more suitable than the traditional Fourier analysis for the merging phenomenon that is not necessarily being periodic in time (e.g., a laminar separation bubble), ${ }^{62}$ and this tool should be preferred for the similar flow in future studies.

\section{Hydraulic characteristics in the mixing layer}

\section{Thickness of the mixing layer}

As a significant velocity gap exists between the groyne field and the main channel, a mixing layer develops along the interface. It is a commonplace to adopt the thickness $\delta$ of the mixing layer to describe its spreading angle and the scope of the large velocity gradient between the two regions. The thickness $\delta(\mathrm{m})$ is defined as follows: $:^{21,28}$

$$
\delta=\frac{U_{m}(x)-U_{e}(x)}{(\partial \bar{u} / \partial y)_{\max }},
$$

where $U_{m}(x)(\mathrm{m} / \mathrm{s})$ and $U_{e}(x)(\mathrm{m} / \mathrm{s})$ are the time-averaged streamwise velocities in the main channel and in the embayment, respectively. Note that the corresponding $y$ coordinates are the locations where the absolute value of the velocity gradient is negligibly small, i.e., lower than $0.5 \mathrm{~s}^{-1}$, with reference to Mignot et al. ${ }^{28}$ In Eq. (8), $(\partial \bar{u} / \partial y)_{\max }\left(\mathrm{s}^{-1}\right)$ represents the maximum velocity gradient at each $x$ position along the interface. However, this approach to determine the thickness does not provide a good reflection of the asymmetry of the time-averaged streamwise velocity profile because there is a sharp decrease in flow velocity near the edge of the vegetation. ${ }^{63}$ Therefore, separating the mixing layer into two parts, namely, an inner layer inside the embayment and an outer layer in the main channel (Fig. 17), appears to be a better approach, similar to that of Truong and Uijttewaal. ${ }^{47}$ Equation (8) is then transformed into the following form:

$$
\delta=\delta_{\text {in }}+\delta_{\text {out }}=\frac{U_{i}(x)-U_{e}(x)}{(\partial \bar{u} / \partial y)_{\max }}+\frac{U_{m}(x)-U_{i}(x)}{(\partial \bar{u} / \partial y)_{\max }},
$$

where $\delta_{\text {in }}(\mathrm{m})$ and $\delta_{\text {out }}(\mathrm{m})$ are the thicknesses of the inner and outer mixing layers and $U_{i}(x)(\mathrm{m} / \mathrm{s})$ is the time-averaged streamwise velocity at the interface.

Figure 18 shows the streamwise evolution of the mixing layer thicknesses $\delta_{\text {in }}$ and $\delta_{\text {out }}$ from the PIV and LES, where the comparison between these two methods is presented for case 1 and it shows 
(a)

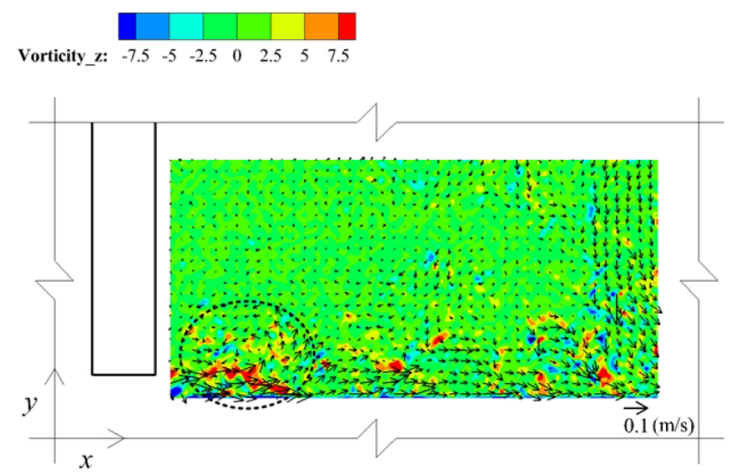

(c)

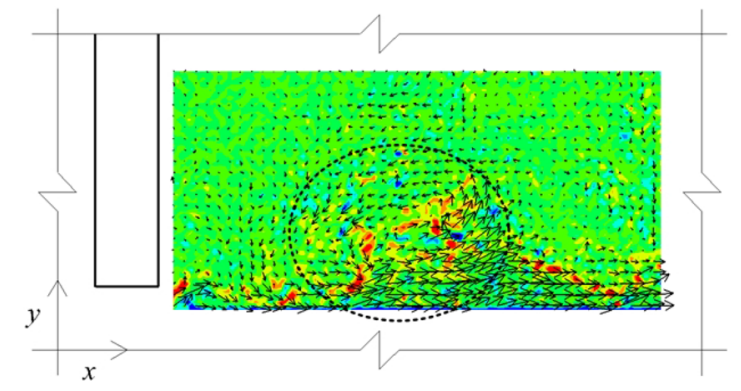

(b)

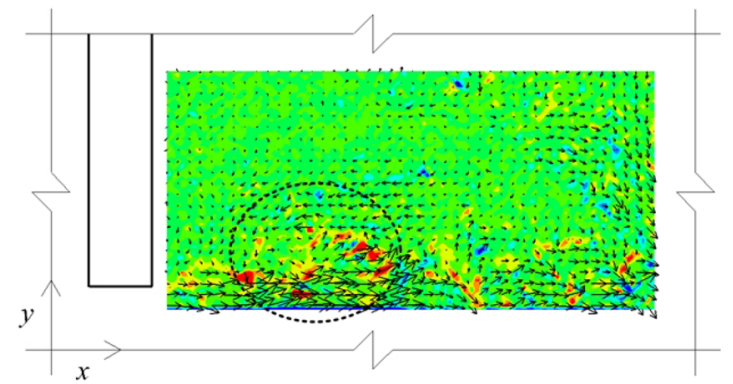

(d)

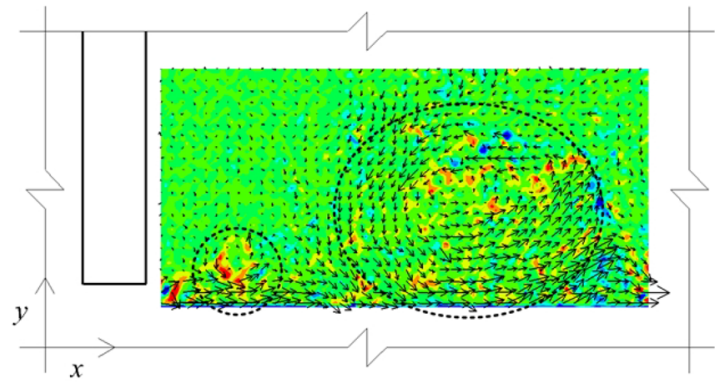

FIG. 13. Development of an eddy shed behind the tip of the upstream groyne at $z=0.625 H$ in case 1 of PIV ( $\Delta t \approx 1 \mathrm{~s}):$ (a) $T_{0}$; (b) $T_{0}+\Delta t$; (c) $T_{0}+2 \Delta t$; and (d) $T_{0}+3 \Delta t$.

(a)

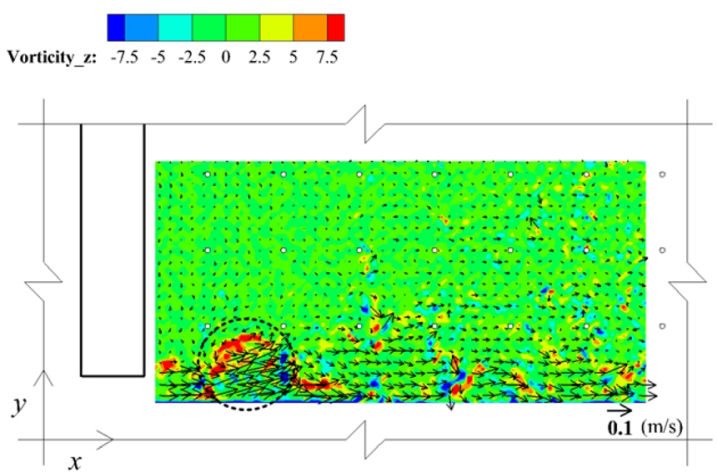

(c)

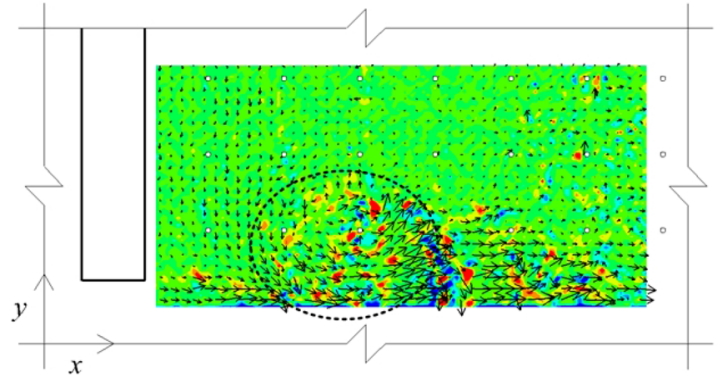

(b)

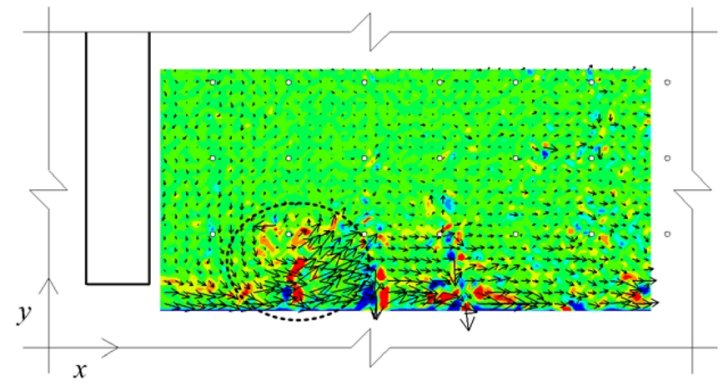

(d)

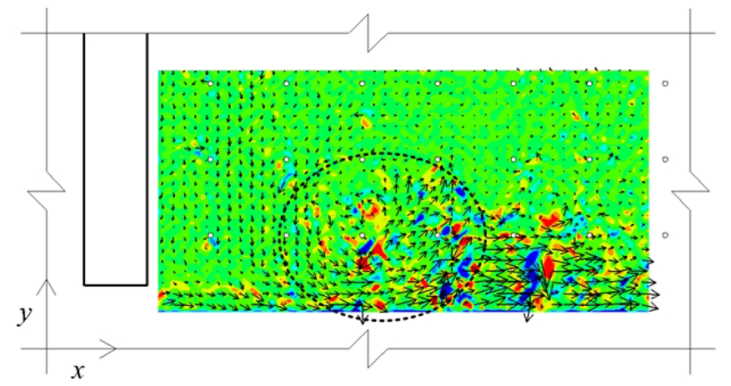

FIG. 14. Development of an eddy shed behind the tip of the upstream groyne at $z=0.625 H$ in case 2 of PIV ( $\Delta t \approx 1 \mathrm{~s})$ : (a) $T_{0}$; (b) $T_{0}+\Delta t$; (c) $T_{0}+2 \Delta t$; and (d) $T_{0}+3 \Delta t$. 
(a)

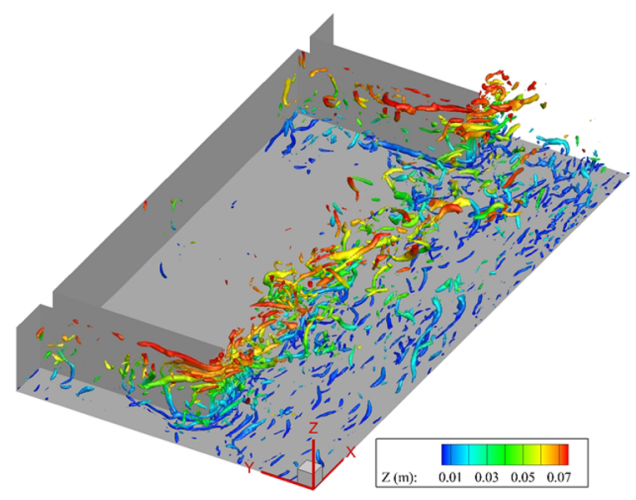

(c)

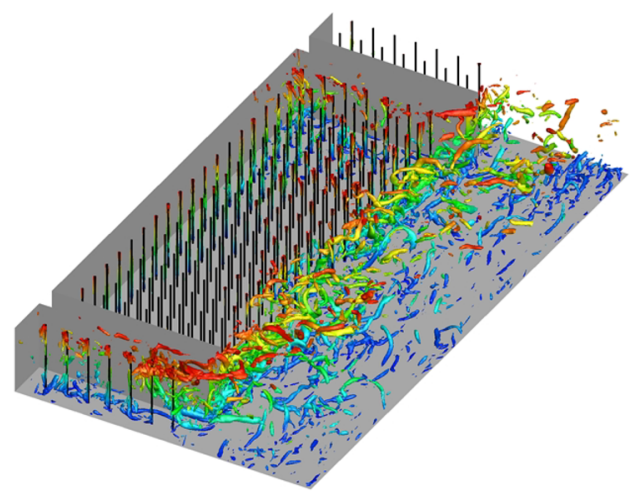

(b)

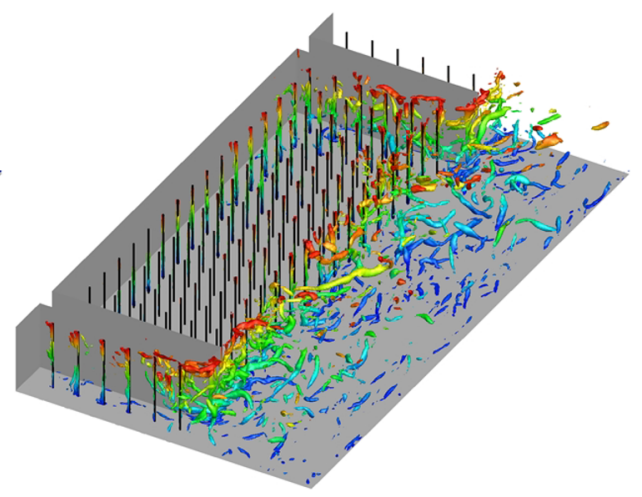

(d)

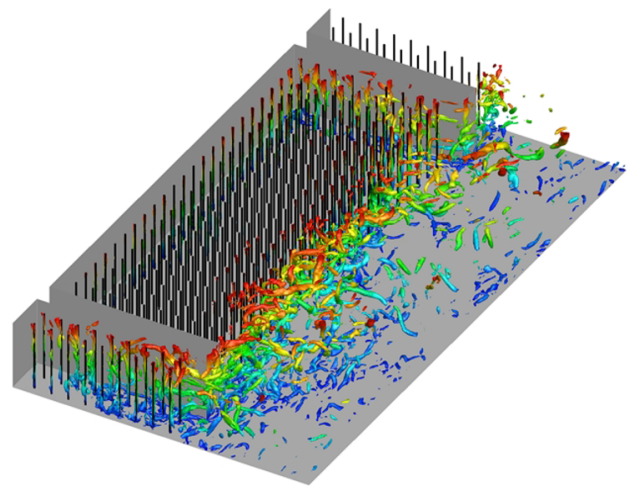

FIG. 15. Instantaneous coherent structures visualized by an iso-surface of $Q=55 \mathrm{~s}^{-2}$ that is colored with the flow depth to better highlight the threedimensionality: (a) case 1 ; (b) case 2; (c) case 3; (d) case 4; and (e) case 5.

(e)

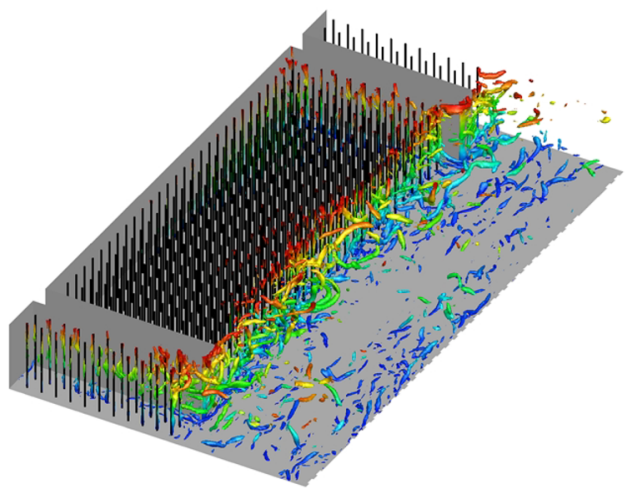

a good agreement (maximum errors are $0.028 W$ and $0.051 W$ for $\delta_{\text {in }}$ and $\delta_{\text {out }}$, respectively).

Overall, the inner thickness $\delta_{\text {in }}$ increases linearly when $x / W$ $<2$ (taking the tip of the upstream groyne as the origin) and then decreases when $x / W>2$ owing to the presence of an adverse pressure gradient near the downstream groyne, which is consistent with the observation of Mignot et al. ${ }^{28}$ The outer thickness $\delta_{\text {out }}$ increases linearly in the upstream part of the mixing layer (i.e., $x / W<1.5$ for case 1 , and $x / W<1$ for cases 3 and 5) and then basically does not change in the downstream part. Note that when the vegetation density increases in the groyne field, the growth rate of the inner thickness $\delta_{\text {in }}$ gradually falls from $k=0.1340$ to $k=0.0682$ [Figs. 18(a), 18(c), and 18(e)]. This is caused by the blocking effect of the vegetation, which inhibits the penetration of the mixing layer and, thus, limits its free growth, as shown in Figs. 13 and 14. The growth rate of the outer mixing layer thickness is nearly constant, $k=0.1662$ -0.1967 [Figs. 18(b), 18(d), and 18(f)], because the outer mixing layer is nearly unaffected by the vegetated region. 
(a)

(b)
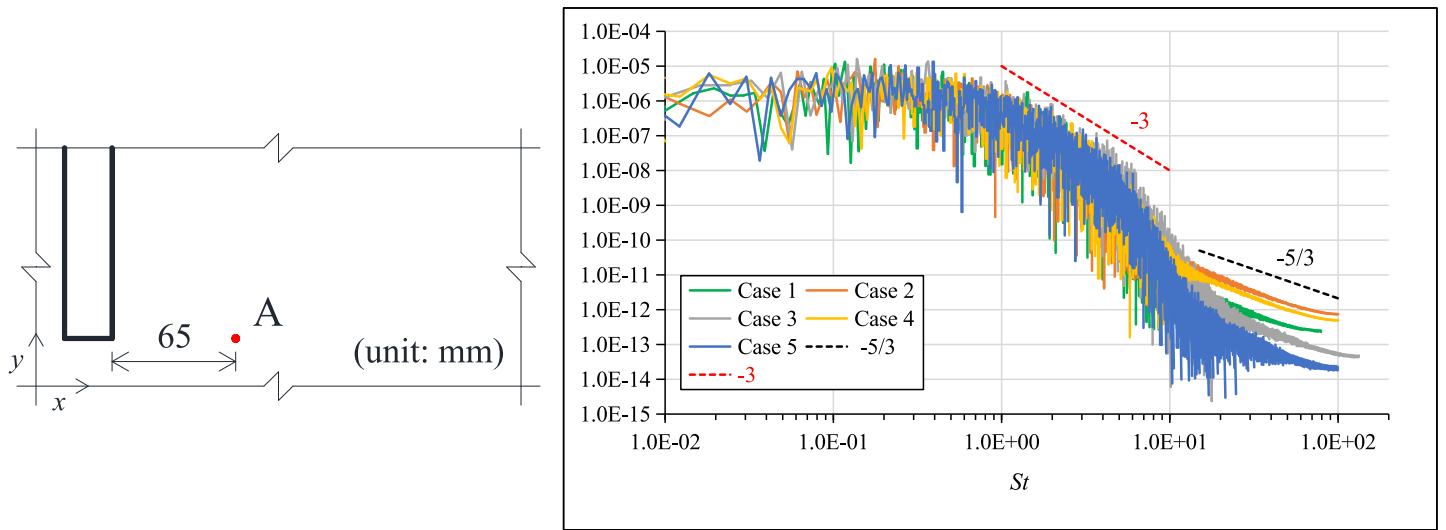

(c)

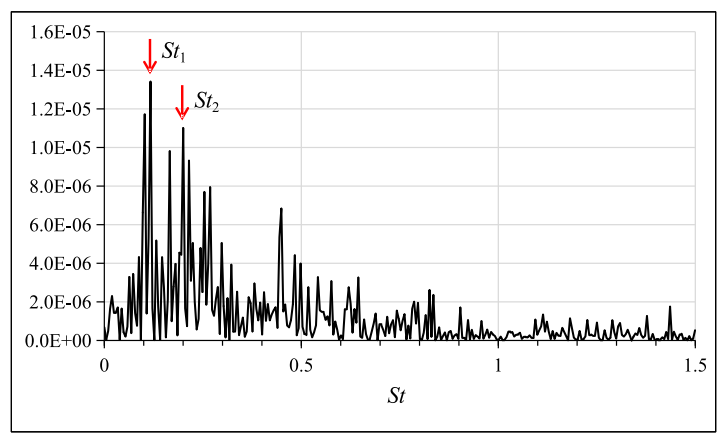

(d)

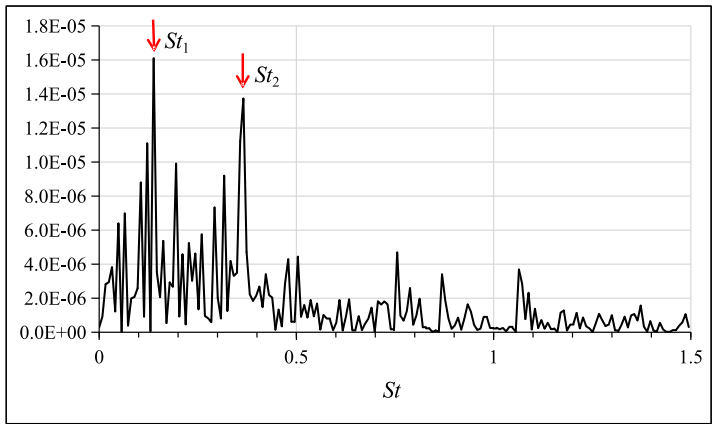

(e)

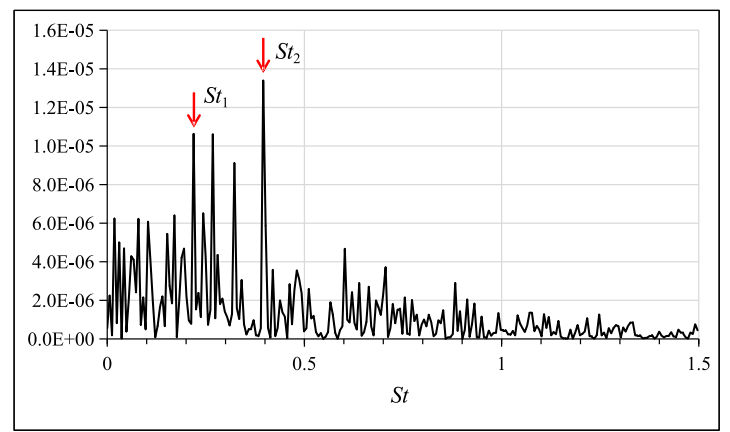

FIG. 16. Power spectra of transverse velocity at point A situated at $z=0.875 \mathrm{H}$ from the LES: (a) sketch of the point $A$; (b) power spectra for the different cases; (c) partial power spectrum for case 1; (d) partial power spectrum for case 3; and (e) partial power spectrum for case 5.

\section{Vorticity}

Figure 19 illustrates the time-averaged vertical vorticity $\Omega_{z}$ (normalized by $U / H$ ) at $z=0.625 H$ from the LES. The $\Omega_{z}$ is defined as follows:

$$
\Omega_{z}=\frac{\partial \bar{v}}{\partial x}-\frac{\partial \bar{u}}{\partial y}
$$

where $\bar{u}$ and $\bar{v}$ represent the mean streamwise velocity and the mean spanwise velocity, respectively.
For all the cases, the mixing layer is permeated with high vorticity owing to the presence of coherent structures. The magnitude of the vorticity decreases in the downstream direction, as proved by the fact that the vorticity in region $\mathrm{A}$ is much greater than in region B. This can be attributed to the evolution of eddies shed from the groyne. Behind the tip of the groyne, small-scale eddies are initially generated by the velocity gradient between the cavity and the main channel. As a result of the significant velocity gap, these eddies have high rates of rotation, and thus, there is a high level of vorticity in the upstream part of region A. As the eddies migrate downstream, 


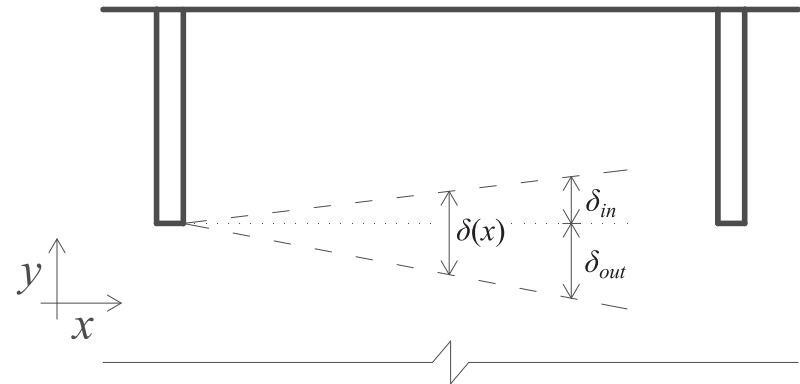

FIG. 17. Division of the mixing layer into inner and outer parts to calculate its thickness.

(a)

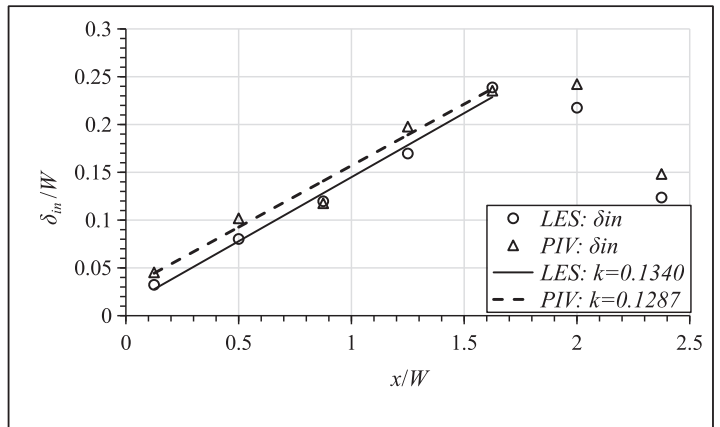

(c)

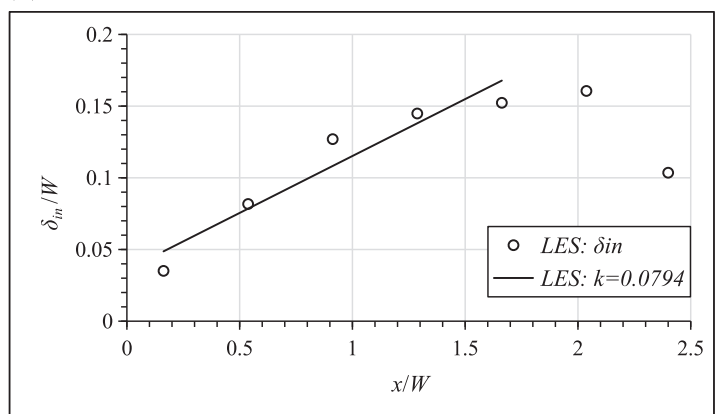

(e)

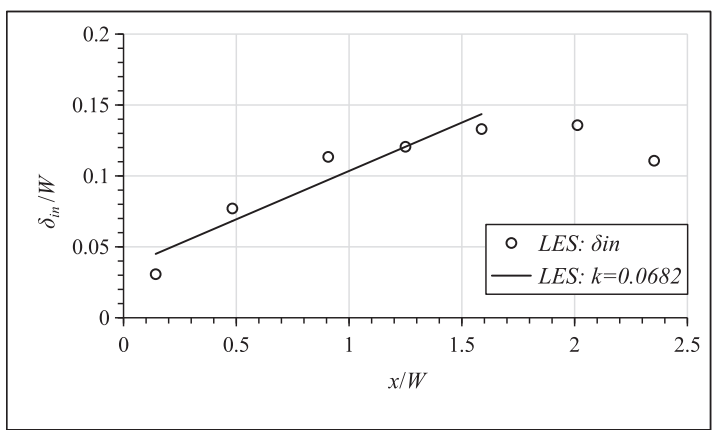

random mergers occur in the mixing layer. Alongside this process, low-vorticity water from the embayment is sucked into the merging eddies, thus leading to a decrease in the total level of vorticity in the downstream part of region $\mathrm{A}$. When the coherent eddies arrive at region $\mathrm{B}$, their vorticity is relatively low, owing to dissipation in the surrounding flow.

A comparison of the vorticity distributions for different cases shows that, in region $B$, the vorticity level in the vegetated cases [Figs. 19(b)-19(e)] is higher than that in the non-vegetation case [Fig. 19(a)]. In the vegetated cases, the presence of plants suppresses the momentum exchange across the interface, and only a small amount of low-vorticity water enters the mixing layer from the groyne field. This ensures that the high level of vorticity

(b)

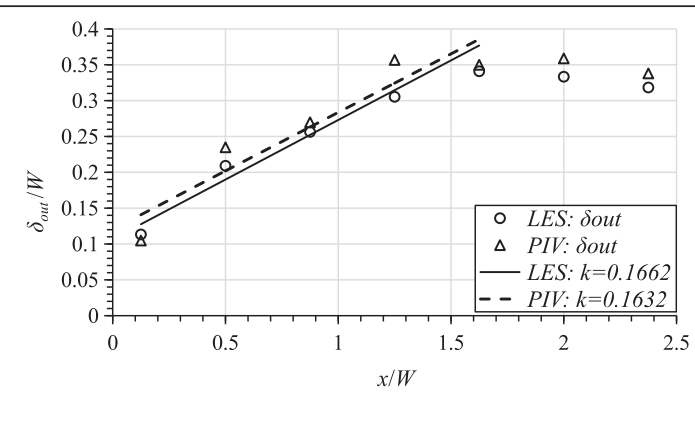

(d)

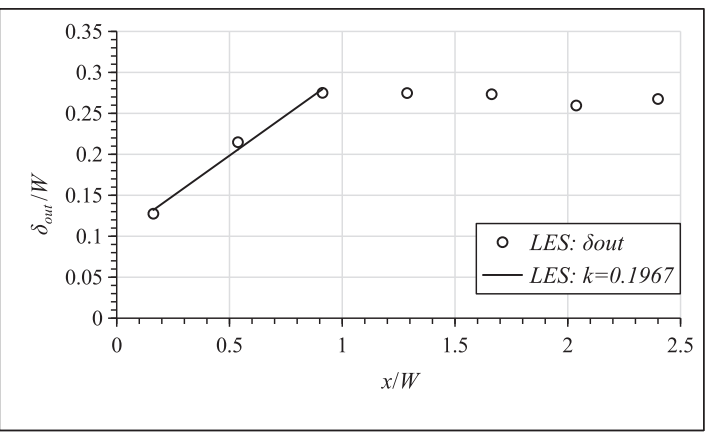

(f)

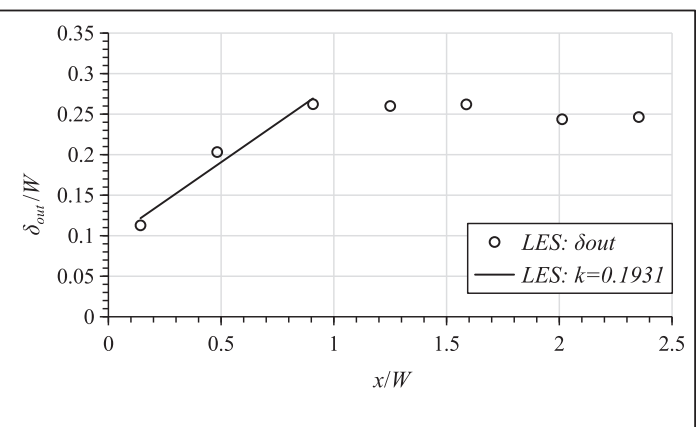

FIG. 18. Streamwise evolution of the mixing layer thicknesses $\delta_{\text {in }}$ and $\delta_{\text {out }}$ at $z=0.625 H$ from the PIV and LES: (a) $\delta_{\text {in }}$ and (b) $\delta_{\text {out }}$ for case 1 ; (c) $\delta_{\text {in }}$ and (d) $\delta_{\text {out }}$ for case 3 ; and $(\mathrm{e}) \delta_{\text {in }}$ and (f) $\delta_{\text {out }}$ for case 5. 
(a)

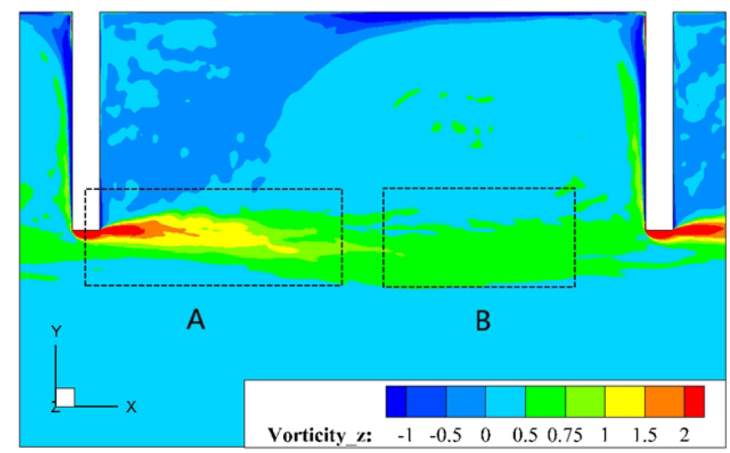

(c)

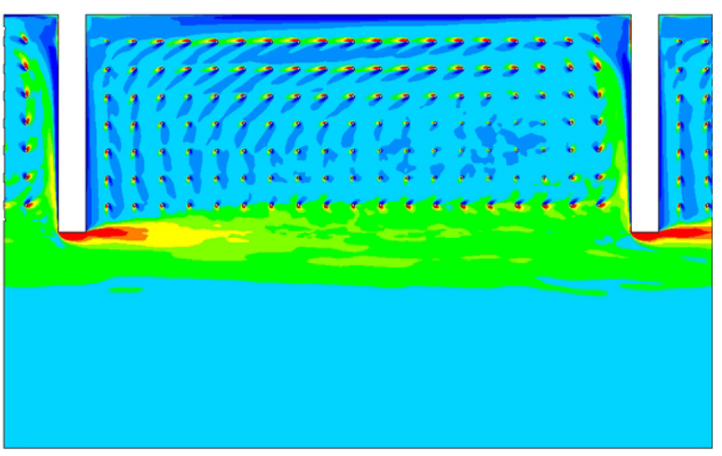

(b)

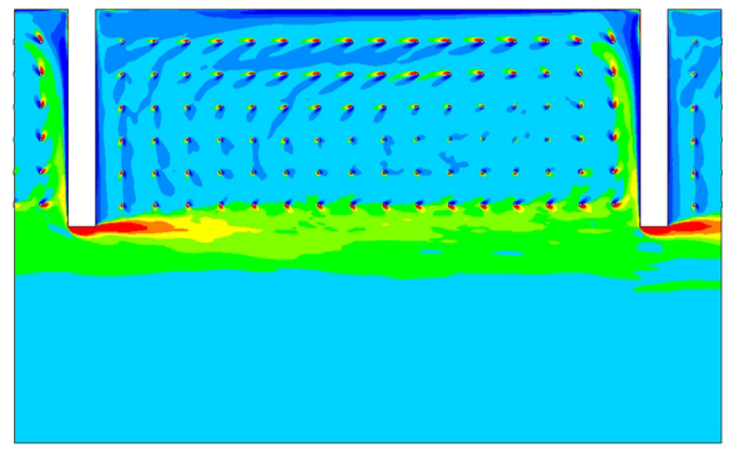

(d)

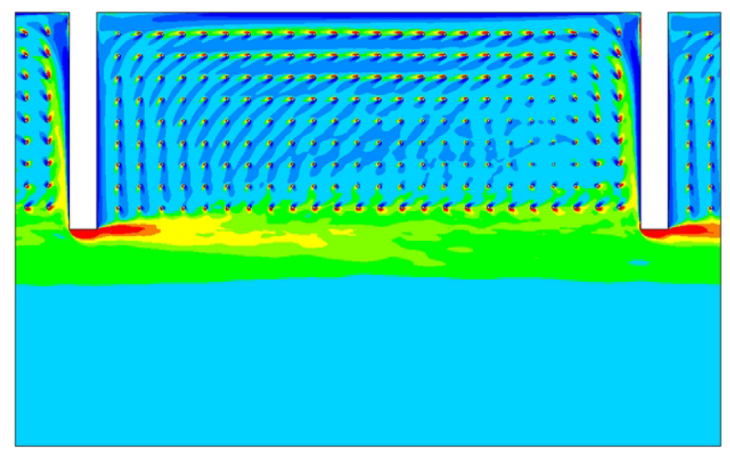

(e)

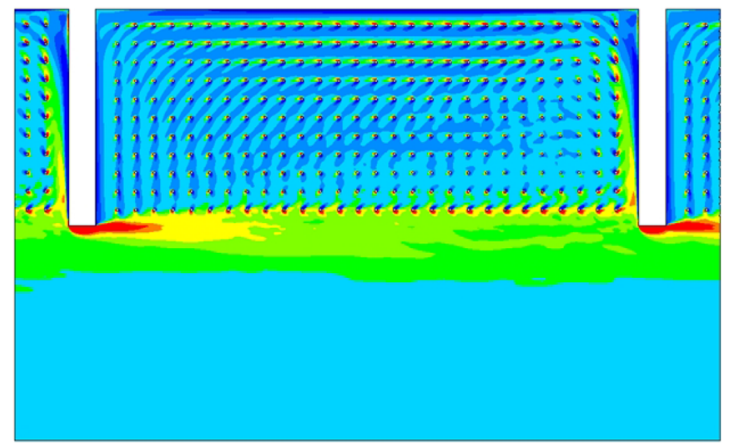

FIG. 19. Time-averaged vorticity at $z=0.625 H$ from the LES: (a) case 1 ; (b) case 2 ; (c) case 3 ; (d) case 4 ; and (e) case 5 .

in the mixing layer is not dissipated rapidly, and thus, the vorticity is greater in the vegetated cases than in the case without vegetation.

\section{Turbulent kinetic energy}

The turbulent kinetic energy (TKE) is defined as follows:

$$
T K E=0.5\left(\overline{u^{\prime 2}}+\overline{v^{\prime 2}}+\overline{w^{\prime 2}}\right),
$$

where the overbar represents the time-averaged value. $u^{\prime}, v^{\prime}$, and $w^{\prime}$ are the velocity fluctuations in the $x$-direction, $y$-direction, and $z$ direction, respectively.

Similar to the vorticity distribution, strong TKE, normalized by $U^{2}$, is concentrated in the mixing layer owing to the instability of the Kelvin-Helmholtz shear layer (see Fig. 20). The level of TKE gradually decreases downstream, as can be seen in regions A and B, due to the dissipation in the surrounding water. When the mergers among the eddies shed from the groyne, water with low TKE is transferred 
(a)

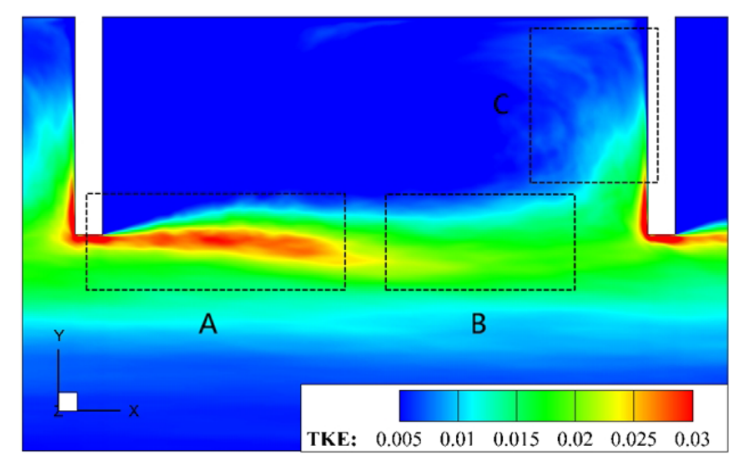

(c)

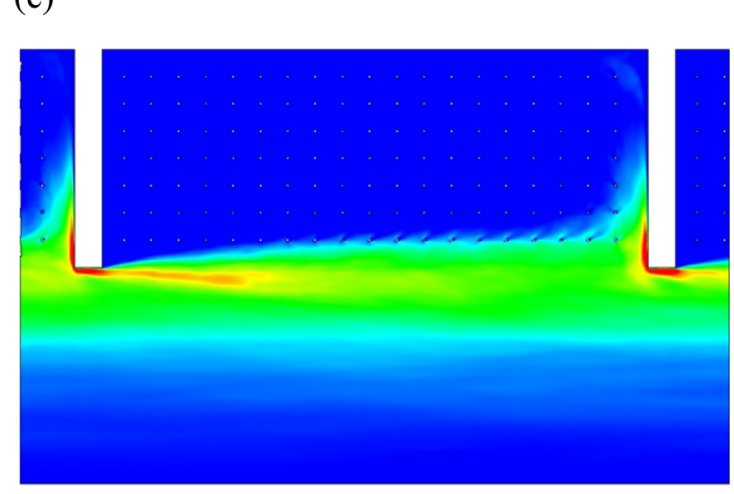

(b)

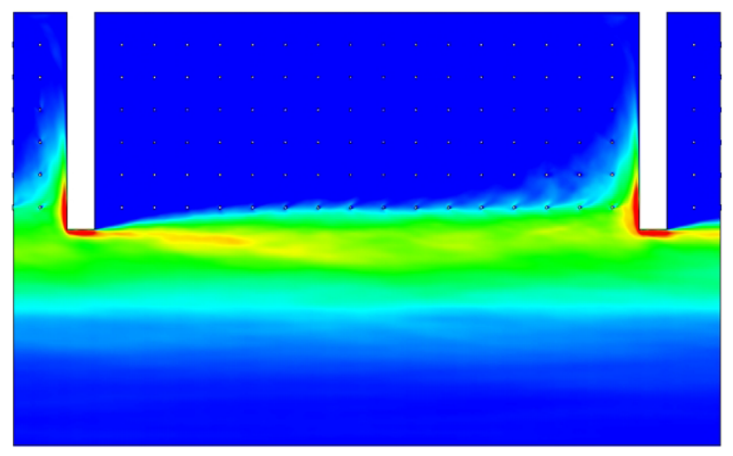

(d)

(e)
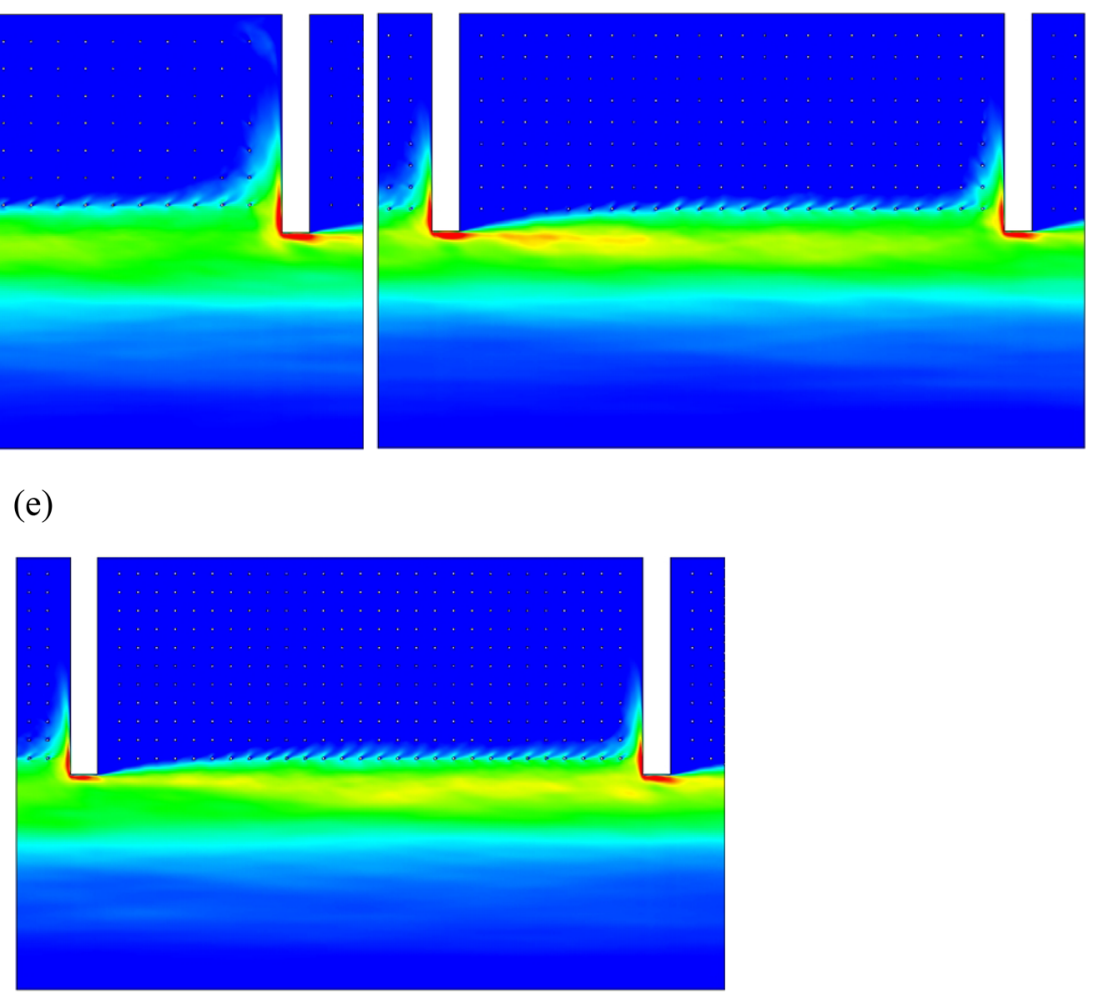

FIG. 20. Time-averaged TKE at $z=0.625 \mathrm{H}$ from the LES: (a) case 1 ; (b) case 2; (c) case 3 ; (d) case 4; and (e) case 5.

from the embayment into the shear layer, leading to a decrease in the turbulent intensity. In region $\mathrm{A}$, the turbulence is more intense in case 1 than in the vegetated cases because the shear layer undergoes much stronger oscillations, not constrained by the edge of the vegetation. The presence of vegetation inhibits water exchange across the interface: both weakly turbulent water from the embayment into the mixing layer and strongly turbulent coherent eddies from the mixing layer into the embayment. This is why the turbulence in the absence of vegetation is lower in region B and higher in region C than in the vegetated cases.

\section{E. Phenomenological model}

Based on the above-mentioned results, a phenomenological model for the evolution of the coherent eddies shed from a groyne is illustrated in Fig. 21. Initially, small-scale eddies A and B are 

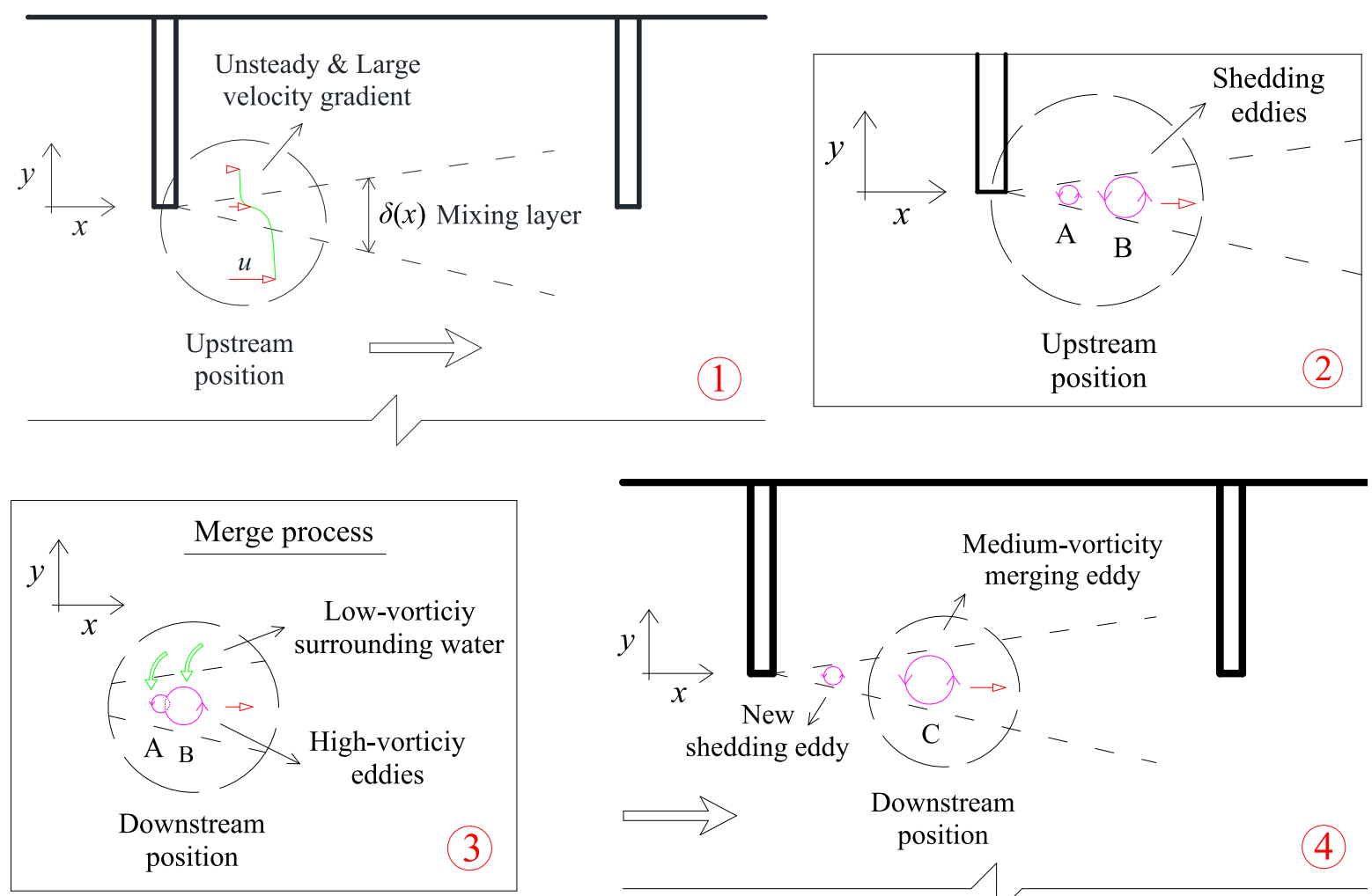

position

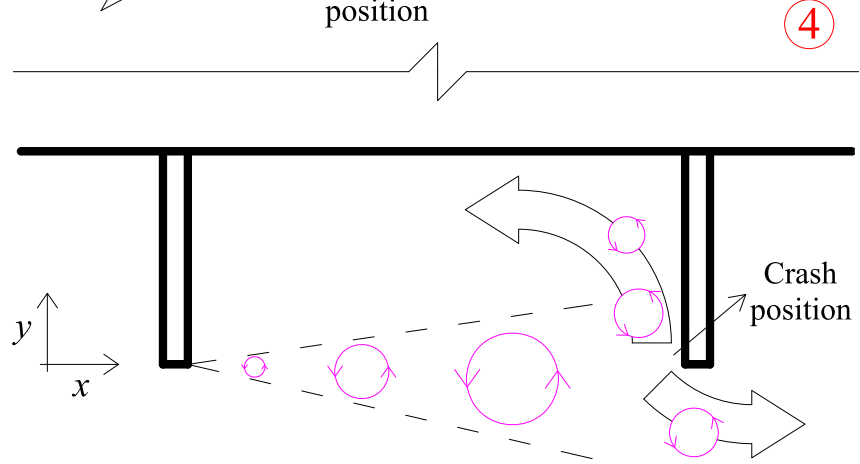

Coherent sructure

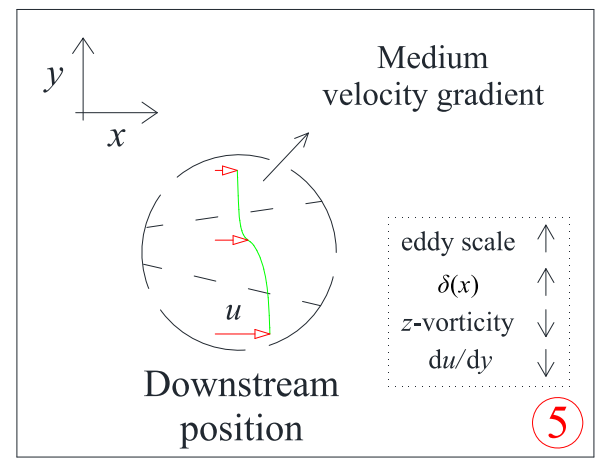

(Kelvin-Helmholtz type vortices)

FIG. 21. Phenomenological model for the evolution of coherent shedding eddies shed from a groyne.

generated behind the tip of the groyne owing to the strong velocity gradient, as shown in panels 1 and 2 (see Fig. 21). Due to the strong mixing in this region, the eddies exhibit high vorticity and strong TKE (see Figs. 19 and 20). When they flow downstream, the adjacent eddies $\mathrm{A}$ and $\mathrm{B}$ merge together through pairing. Accompanied by this process, the surrounding water of low vorticity and TKE is sucked from the groyne field into the mixing layer, as shown in panel 3. After sufficient mixing, a large-scale merged eddy $\mathrm{C}$ is formed, concomitantly with the increase in the mixing layer width, as shown in panel 4. In addition, a newly shedding eddy of a small size is produced behind the groyne, which will follow the similar evolution to eddies A and B. Due to the dissipation in the surrounding water, the hydrodynamic parameters involved in the merged eddy $\mathrm{C}$ exhibit lower values, i.e., the vorticity, velocity gradient, and TKE all decrease, as shown in panel 5. The shedding and the merger of eddies occur periodically, and thus, a large-scale coherent structure is eventually formed in the mixing layer, as shown in panel 6. After crashing into the downstream groyne, some of these coherent eddies enter the groyne field in the form of a wall-attached jet-like flow, while others bypass the tip of the groyne and flow downstream. In 
the vegetated cases, the growth of the mixing layer is limited by the edge of vegetation patches, and the hydrodynamic parameters in the mixing layer are relatively large due to the blocking effect of the vegetation.

\section{F. Discussion}

Referring to the previous studies, ${ }^{11,13-15,17,21,31,32}$ the present experiment is carried out in an idealized testing model, i.e., the straight fixed-bed channel of a rectangular cross section with regular groynes on one side, which could reflect basic flowing features in natural groyne fields (e.g., the circulation structure, the KelvinHelmholtz shear layer, and the vortex shedding phenomenon). With the presence of vegetation, the reduction in the circulation velocity and turbulent intensity could promote sediment deposition, the decrease in the exchange coefficient could extend the retention process of organic matters inside groyne fields, and the increase in hydraulic properties of the mixing layer could improve the dynamic level near the cavity mouth. All these influences are beneficial to stream corridor restoration: for example, the vegetation zone could act as breeding grounds for fish (increasing the bio-diversity of river systems), the sedimentation process could enhance the inhomogeneity of river morphology (creating diverse environments for aquatic organism), the long residence time could cause dissolved nutrients to be absorbed adequately by plants (improving water quality), and the relative high dynamic level could prevent the accumulation of sediments in the cavity mouth (maintaining the hydrological connectivity between the cavity and the main-stream). The above-mentioned knowledge can help river managers understand vegetation effects on flow hydrodynamics and realize the ecological function of vegetated groyne fields more deeply.

However, due to the difference between realistic situations and idealized experiment conditions, the above-mentioned results could not be exactly the same as those in natural groyne fields. In reality, rivers are always meandering, ${ }^{64}$ and this could cause groynes to be inclined against the flow direction (i.e., not perpendicular to the main-stream), which leads to the change in the flow behavior: for example, in cases of backward inclined groynes, the size of the secondary gyre gets larger and the retention time inside the cavity becomes longer, ${ }^{51,65}$ and in forward inclined cases, the groyne increases the secondary flow cell along the stream bank, thus resulting in the displacement of channel thalweg toward the channel center. $^{66}$ In addition, natural compound channels could lead to a lower mean water depth in the cavity than in the main-stream, which enhances the bottom friction and reduces the exchange coefficient. ${ }^{15,65}$ Furthermore, natural flow belongs to the multi-phase material, i.e., the mixture of air, water, and sand. The testing flow, here, is just set as clean water, which deviates from the realistic situation to some extent. The interaction between liquid and solid phases does have an effect on the flow property, for example, suspend sediments could reduce the turbulence, ${ }^{67}$ particle-coherent structure interactions might affect vortex shedding processes, ${ }^{68}$ and the scoured hole could adjust the distribution of hydraulic characteristics around groynes. Therefore, the next stage of this research should focus on near-natural cases (e.g., compound channel, sediments, and mobile-bed), which is a better way to study the turbulent flow in groyne fields.

\section{CONCLUSIONS}

In the absence of sediments, the hydrodynamics of consecutive groyne fields with different vegetation densities have been investigated both experimentally through PIV and numerically through the LES. The results reveal that the vegetation causes the double gyres (non-vegetation case) to be transformed into a single gyre, with a reduction in the circulation velocity. This is attributed to a combination of the blocking effect of vegetation and its drag force. In the main flow, a pair of streamwise secondary currents is present. For the internal secondary current, it is too feeble to generate the vertical flow in the cavity effectively.

As the vegetation suppresses momentum exchange between the cavity and the main channel, the exchange coefficient gradually decreases with an increasing vegetation density. It should be emphasized that the exchange rate is obtained from a newly proposed exchange layer at a distance of $0.04 \mathrm{~W}$ from the groyne tip (where $W$ is the length of the groyne). This location is considered to be a more appropriate choice than the interface, since it prevents the flow bypassing the tip of the groyne from the exchange process.

Small-scale eddies are shed from the tip of the groyne due to the unsteady velocity gradient. As they flow downstream, these eddies merge together to form a relatively large-scale eddy, resulting in an increase in the mixing layer width. The eddy shedding and merger process occur in a periodic manner, eventually resulting in the formation of large-scale coherent structures along the interface. It should be noted that as the vegetation density increases, both the master frequency of eddy shedding and the dominant frequency of the merged eddies gradually increase. The hydrodynamic parameters corresponding to the coherent structures, namely, the vorticity and the turbulent kinetic energy, both decrease in the downstream direction. In the downstream part of the shear layer, their values are higher for the vegetated cases than in the absence of vegetation, which is attributable to the reduced inflow from the embayment into the merging eddies.

Based on the above-mentioned results, a phenomenological model is presented to describe the evolution of the coherent structures and the variations in flow hydrodynamics associated with these eddies. This model should be useful to clarify the flow characteristics of the mixing layer in the consecutive vegetated groyne fields.

This study enriches the knowledge on the interaction between aquatic vegetation and circulation flow in groyne fields, and plant communities are thought to play a positive role in stream corridor restoration. With the presence of vegetation, the sediment deposition could be enhanced, concomitantly with the diversity of river morphology, and the retention process of organic materials is extended, which is beneficial to the absorption and purification process of an aquatic organism. In addition, the vegetation leads to a relatively high dynamic level near the cavity mouth, thus maintaining the hydrological connectivity between the groyne field and the main-stream.

\section{ACKNOWLEDGMENTS}

The authors gratefully acknowledge the financial support from the National Natural Science Foundation of China (Grant Nos. 51679170 and 51879199) and the Major Technology Innovation of Hubei Province (Grant No. 2019ACA154). The authors also thank 
editors and anonymous reviewers for their helpful comments on this paper.

\section{DATA AVAILABILITY}

The data that support the findings of this study are available from the corresponding author upon reasonable request.

\section{REFERENCES}

${ }^{1}$ M. F. Yossef, "The effect of groynes on rivers: Literature review," Delft Cluster Publicatienummer. 03.03. 04, 2002.

${ }^{2} \mathrm{H}$. King, "The use of groynes for riverbank erosion protection," University of Stellenbosch CPD course "River hydraulics, stormwater and flood management, 2009.

${ }^{3}$ B. Przedwojski, "Bed topography and local scour in rivers with banks protected by groynes," J. Hydraul, Res. 33, 257-273 (1995).

${ }^{4}$ A. N. Sukhodolov, "Hydrodynamics of groyne fields in a straight river reach: Insight from field experiments," J. Hydraul. Res. 52(1), 105-120 (2014).

${ }^{5}$ A. Sukhodolov, C. Engelhardt, A. Krüger, and H. Bungartz, "Case study: Turbulent flow and sediment distributions in a groyne field," J. Hydraul. Eng. 130(1), $1-9$ (2004).

${ }^{6} \mathrm{~J}$. G. Duan, L. He, X. Fu, and Q. Wang, "Mean flow and turbulence around experimental spur dike," Adv. Water Resour. 32(12), 1717-1725 (2009).

${ }^{7}$ J. Qin, D. Zhong, T. Wu, and L. Wu, "Sediment exchange between groin fields and main-stream," Adv. Water Resour. 108, 44-54 (2017).

${ }^{8}$ M. F. Yossef and H. J. de Vriend, "Sediment exchange between a river and its groyne fields: Mobile-bed experiment," J. Hydraul. Eng. 136(9), 610-625 (2010).

${ }^{9}$ C. Engelhardt, A. Krüger, A. Sukhodolov, and A. Nicklisch, "A study of phytoplankton spatial distributions, flow structure and characteristics of mixing in a river reach with groynes," J. Plankton Res. 26(11), 1351-1366 (2004).

${ }^{10} \mathrm{P}$. Aziz and A. Kadota, "Experimental study of morphological changes and flow structure around the vegetated groyne," Int. J. Adv. Sci., Eng. Inf. Technol. 8(1), 99-107 (2018).

${ }^{11}$ W. Brevis, M. García-Villalba, and Y. Niño, "Experimental and large eddy simulation study of the flow developed by a sequence of lateral obstacles," Environ. Fluid Mech. 14(4), 873-893 (2014).

${ }^{12}$ H. Fang, J. Bai, G. He, and H. Zhao, "Calculations of nonsubmerged groin flow in a shallow open channel by large-eddy simulation," J. Eng. Mech. 140(5), 04014016 (2014).

${ }^{13}$ E. Mignot, W. Cai, and N. Rivière, "Analysis of the transitions between flow patterns in open-channel lateral cavities with increasing aspect ratio," Environ. Fluid Mech. 19(1), 231-253 (2019).

${ }^{14} \mathrm{M}$. Sanjou and I. Nezu, "Fundamental study on mixing layer and horizontal circulation in open-channel flows with rectangular embayment zone," J. Hydrodyn. 29(1), 75-88 (2017).

${ }^{15}$ W. S. J. Uijttewaal, D. V. Lehmann, and A. V. Mazijk, "Exchange processes between a river and its groyne fields: Model experiments," J. Hydraul. Eng. 127(11), 928-936 (2001)

${ }^{16}$ A. McCoy, G. Constantinescu, and L. J. Weber, "Numerical investigation of flow hydrodynamics in a channel with a series of groynes," J. Hydraul. Eng. 134(2), 157-172 (2008).

${ }^{17}$ Y. Akutina, S. J. Gaskin, and L. B. Mydlarski, "Entrainment mechanisms in river embayments," in River Flow (Taylor \& Francis Group, 2014), pp. 31-37.

${ }^{18} \mathrm{~K}$. Xiang, Z. Yang, W. Huai, and R. Ding, "Large eddy simulation of turbulent flow structure in a rectangular embayment zone with different population densities of vegetation,” Environ. Sci. Pollut. Res. 26(14), 14583-14597 (2019).

${ }^{19} \mathrm{~A}$. McCoy, G. Constantinescu, and L. Weber, "Exchange processes in a channel with two vertical emerged obstructions," Flow, Turbul. Combust. 77(1-4), 97-126 (2006).

${ }^{20}$ J. Paik and F. Sotiropoulos, "Coherent structure dynamics upstream of a long rectangular block at the side of a large aspect ratio channel," Phys. Fluids 17(11), 115104 (2005)
${ }^{21}$ M. F. Yossef and H. J. de Vriend, "Flow details near river groynes: Experimental investigation," J. Hydraul. Eng. 137(5), 504-516 (2011).

${ }^{22}$ C. Perrot-Minot, E. Mignot, N. Riviere, and R. Perkins, "Predicting the vortex shedding frequency at the interface of the lateral cavities," in Proceedings of River Flow 2018: 9th International Conference on Fluvial Hydraulics, 40. Lyon, France: E3S Web of Conferences, 2018.

${ }^{23}$ M. Y. Lam, M. S. Ghidaoui, and A. A. Kolyshkin, "The roll-up and merging of coherent structures in shallow mixing layers," Phys. Fluids 28(9), 094103 (2016).

${ }^{24} \mathrm{X}$. Ma and A. Schroder, "Analysis of flapping motion of reattaching shear layer behind a two-dimensional backward-facing step," Phys. Fluids 29(11), 115104 (2017).

${ }^{25}$ K. Takamure, Y. Ito, Y. Sakai, K. Iwano, and T. Hayase, "Momentum transport process in the quasi self-similar region of free shear mixing layer," Phys. Fluids 30(1), 015109 (2018).

${ }^{26}$ G. L. Brown and A. Roshko, "On density effects and large structure in turbulent mixing layers,” J. Fluid Mech. 64(4), 775-816 (1974).

${ }^{27}$ G. Constantinescu, A. Sukhodolov, and A. McCoy, "Mass exchange in a shallow channel flow with a series of groynes: LES study and comparison with laboratory and field experiments," Environ. Fluid Mech. 9(6), 587 (2009).

${ }^{28}$ E. Mignot, W. Cai, G. Launay, N. Riviere, and C. Escauriaza, "Coherent turbulent structures at the mixing-interface of a square open-channel lateral cavity," Phys. Fluids 28(4), 045104 (2016).

${ }^{29}$ J. Sandoval, E. Mignot, L. Mao, P. A. Pastén, D. Bolster, and C. Escauriaza, "Field and numerical investigation of transport mechanisms in a surface storage zone," J. Geophys. Res. 124(4), 938-959, https://doi.org/10.1029/2018jf004716 (2019).

${ }^{30}$ M. Sanjou, T. Akimoto, and T. Okamoto, "Three-dimensional turbulence structure of rectangular side-cavity zone in open-channel streams," Int. J. River Basin Manage. 10(4), 293-305 (2012).

${ }^{31}$ M. Sanjou and I. Nezu, "Hydrodynamic characteristics and related masstransfer properties in open-channel flows with rectangular embayment zone," Environ. Fluid Mech. 13(6), 527-555 (2013).

${ }^{32}$ V. Weitbrecht, S. A. Socolofsky, and G. H. Jirka, "Experiments on mass exchange between groin fields and main stream in rivers," J. Hydraul. Eng. 134(2), 173-183 (2008).

${ }^{33}$ E. Mignot and W. Brevis, "Coherent turbulent structures within open-channel lateral cavities,” J. Hydraul. Eng. 146(2), 04019066 (2020).

${ }^{34} \mathrm{H}$. Liu and Y. Lu, "Experiment of hydraulic structure s effect on pollutant transport and dispersion," 2013 the International Conference on Remote Sensing, Environment and Transportation Engineering (RSETE 2013), 2013.

${ }^{35}$ H. M. Nepf, "Hydrodynamics of vegetated channels," J. Hydraul. Res. 50(3), 262-279 (2012).

${ }^{36}$ T.-a. Okamoto, I. Nezu, and H. Ikeda, "Vertical mass and momentum transport in open-channel flows with submerged vegetations," J. Hydro-environ. Res. 6(4), 287-297 (2012).

${ }^{37}$ A. N. Sukhodolov and T. A. Sukhodolova, "Field experiments in vegetated groyne fields," in Proceedings of 7th International Conference on Fluvial Hydraulics (Taylor \& Francis Group, 2014), pp. 475-481.

${ }^{38}$ A. N. Sukhodolov, T. A. Sukhodolova, and J. Krick, "Effects of vegetation on turbulent flow structure in groyne fields," J. Hydraul. Res. 55(1), 1-15 (2017).

${ }^{39}$ W.-x. Huai, Z.-b. Chen, J. Han, L.-x. Zhang, and Y.-h. Zeng, "Mathematical model for the flow with submerged and emerged rigid vegetation," J. Hydrodyn. 21(5), 722-729 (2009).

${ }^{40} \mathrm{Y}$. Li, L. Xie, and T. Su, "Bio-capture of solid pollutants by vegetation canopy cave in shallow water flow," Int. J. Environ. Res. Public Health 16(23), 4846 (2019).

${ }^{41}$ N. Luminari, C. Airiau, and A. Bottaro, "Drag-model sensitivity of KelvinHelmholtz waves in canopy flows," Phys. Fluids 28(12), 124103 (2016).

${ }^{42} \mathrm{M}$. Sanjou, T. Okamoto, and I. Nezu, "Dissolved oxygen transfer into a square embayment connected to an open-channel flow," Int. J. Heat Mass Transfer 125, 1169-1180 (2018).

${ }^{43}$ A. Monti, M. Omidyeganeh, and A. Pinelli, "Large-eddy simulation of an openchannel flow bounded by a semi-dense rigid filamentous canopy: Scaling and flow structure," Phys. Fluids 31(6), 065108 (2019). 
${ }^{44}$ W. Chang, G. Constantinescu, and W. Tsai, "Effect of array submergence on flow and coherent structures through and around a circular array of rigid vertical cylinders," Phys. Fluids 32(3), 035110 (2020).

${ }^{45} \mathrm{~J}$. Lu and H. C. Dai, "Large eddy simulation of flow and mass exchange in an embayment with or without vegetation," Appl. Math. Modell. 40(17-18), 77517767 (2016)

${ }^{46}$ M. Sanjou and I. Nezu, "Large eddy simulation of compound open-channel flows with emergent vegetation near the floodplain edge," J. Hydrodyn. 22(5), 565 (2010).

${ }^{47}$ S. H. Truong and W. S. J. Uijttewaal, "Transverse momentum exchange induced by large coherent structures in a vegetated compound channel," Water Resour. Res. 55(1), 589-612, https://doi.org/10.1029/2018wr023273 (2019).

${ }^{48}$ W. Huai, W. Xue, and Z. Qian, "Large-eddy simulation of turbulent rectangular open-channel flow with an emergent rigid vegetation patch," Adv. Water Res. 80, 30-42 (2015).

${ }^{49}$ E. Mignot, W. Cai, J. I. Polanco, C. Escauriaza, and N. Riviere, "Measurement of mass exchange processes and coefficients in a simplified open-channel lateral cavity connected to a main stream," Environ. Fluid Mech. 17(3), 429-448 (2017).

${ }^{50}$ V. Weitbrecht, G. Kühn, and G. H. Jirka, "Large scale PIV-measurements at the surface of shallow water flows," Flow Meas. Instrum. 13(5-6), 237-245 (2002).

${ }^{51}$ T. R. Jackson, S. V. Apte, R. Haggerty, and R. Budwig, "Flow structure and mean residence times of lateral cavities in open channel flows: Influence of bed roughness and shape," Environ. Fluid Mech. 15(5), 1069-1100 (2015).

${ }^{52}$ M. Germano, U. Piomelli, P. Moin, and W. H. Cabot, "A dynamic subgrid-scale eddy viscosity model,” Phys. Fluids A 3(7), 1760-1765 (1991).

${ }^{53}$ J. Smagorinsky, "General circulation experiments with the primitive equations," Mon. Weather Rev. 91(3), 99-164 (1963).

${ }^{54}$ M. Gao, W. Huai, Y. Xiao, Z. Yang, and B. Ji, "Large eddy simulation of a vertical buoyant jet in a vegetated channel," Int. J. Heat Fluid Flow 70, 114-124 (2018).

${ }^{55}$ C. Hinterberger, J. Fröhlich, and W. Rodi, "Three-dimensional and depthaveraged large-eddy simulations of some shallow water flows," J. Hydraul. Eng. 133(8), 857-872 (2007).
${ }^{56}$ B. J. Alfrink and L. C. Van Rijn, "Two-equation turbulence model for flow in trenches," J. Hydraul. Eng. 109(7), 941-958 (1983).

${ }^{57}$ W. Gao, W. Zhang, W. Cheng, and R. Samtaney, "Wall-modelled large-eddy simulation of turbulent flow past airfoils," J. Fluid Mech. 873, 174-210 (2019).

${ }^{58}$ C. Gualtieri, "Numerical simulation of mass exchange processes in a dead zone of a river," Advances in Environmental Fluid Mechanics (World Scientific, 2010), Chap. 12.

${ }^{59} \mathrm{M}$. Pusch and H. Fischer, Stoffdynamik und Habitatstruktur in der Elbe (Weissensee Verlag, Berlin, 2006).

${ }^{60}$ E. M. Valentine and I. R. Wood, "Experiments in longitudinal dispersion with dead zones,” J. Hydraul. Div. 105(8), 999-1016 (1979).

${ }^{61}$ V. Weitbrecht, and G. H. Jirka, "Flow patterns in dead zones of rivers and their effect on exchange processes," in Proceedings of the 2001 International Symposium on Environmental Hydraulics, 2001.

${ }^{62}$ J. W. Kurelek, S. Yarusevych, and M. Kotsonis, "Vortex merging in a laminar separation bubble under natural and forced conditions," Phys. Rev. Fluids 4(6), 063903 (2019).

${ }^{63}$ B. L. White and H. M. Nepf, "Shear instability and coherent structures in shallow flow adjacent to a porous layer," J. Fluid Mech. 593, 1-32 (2007).

${ }^{64}$ S. Z. Ali and S. Dey, "Hydrodynamic instability of meandering channels," Phys. Fluids 29(12), 125107 (2017).

${ }^{65} \mathrm{~V}$. Weitbrecht, "Influence of dead-water zones on the dispersive mass transport in rivers," Ph.D. thesis, University of Karlsruhe, Germany, 2004.

${ }^{66} \mathrm{~S}$. Kang and F. Sotiropoulos, "Numerical study of flow dynamics around a stream restoration structure in a meandering channel," J. Hydraul. Res. 53(2), 178-185 (2015).

${ }^{67}$ D. Cloutier, M. N. LeCouturier, C. L. Amos, and P. R. Hill, "The effects of suspended sediment concentration on turbulence in an annular flume," Aquat. Ecol. 40(4), 555-565 (2006).

${ }^{68}$ J. L. Best, S. Bennett, J. Bridge, and M. Leeder, “Turbulence modulation and particle velocities over flat sand beds at low transport rates," J. Hydraul. Eng. 123(12), 1118-1129 (1997). 